\title{
The Probability That A Numerical Analysis Problem Is Difficult
}

\author{
By James W. Demmel
}

\begin{abstract}
Numerous problems in numerical analysis, including matrix inversion, eigenvalue calculations and polynomial zerofinding, share the following property: The difficulty of solving a given problem is large when the distance from that problem to the nearest "ill-posed" one is small. For example, the closer a matrix is to the set of noninvertible matrices, the larger its condition number with respect to inversion. We show that the sets of ill-posed problems for matrix inversion, eigenproblems, and polynomial zerofinding all have a common algebraic and geometric structure which lets us compute the probability distribution of the distance from a "random" problem to the set. From this probability distribution we derive, for example, the distribution of the condition number of a random matrix. We examine the relevance of this theory to the analysis and construction of numerical algorithms destined to be run in finite precision arithmetic.
\end{abstract}

1. Introduction. To investigate the probability that a numerical analysis problem is difficult, we need to do three things:

(1) Choose a measure of difficulty,

(2) Choose a probability distribution on the set of problems,

(3) Compute the distribution of the measure of difficulty induced by the distribution on the set of problems.

The measure of difficulty we shall use in this paper is the condition number, which measures the sensitivity of the solution to small changes in the problem. For the problems we consider in this paper (matrix inversion, polynomial zerofinding and eigenvalue calculation), there are well-known condition numbers in the literature of which we shall use slightly modified versions to be discussed more fully later. The condition number is an appropriate measure of difficulty because it can be used to measure the expected loss of accuracy in the computed solution, or even the number of iterations required for an iterative algorithm to converge to a solution.

The probability distribution on the set of problems for which we will attain most of our results will be the "uniform distribution" which we define as follows. We will identify each problem as a point in either $\mathbf{R}^{N}$ (if it is real) or $\mathbf{C}^{N}$ (if it is complex). For example, a real $n$ by $n$ matrix $A$ will be considered to be a point in $\mathbf{R}^{n^{2}}$, where each entry of $A$ forms a coordinate in $\mathbf{R}^{n^{2}}$ in the natural way. Similarly, a complex $n$th degree polynomial can be identified with a point in $\mathbf{C}^{n+1}$ by using its coefficients as coordinates. On the space $\mathbf{R}^{N}$ (or $\mathbf{C}^{N}$ ) we will take any spherically symmetric distribution, i.e., the induced distribution of the normalized problem $x /\|x\|(\|\cdot\|$ is the Euclidean norm) must be uniform on the unit sphere in

Received May 4, 1987; revised August 18, 1987.

1980 Mathematics Subject Classification (1985 Revision). Primary 15A12, 53C65, 60D05.

Parts of this paper appeared previously under the title "The Geometry of Ill-Conditioning," in The Journal of Complexity, Vol. III, pgs. 201-229, 1987. Copyright (C) 1987 Academic Press, Inc. Reprinted by permission of the publisher. 
$\mathbf{R}^{N}$. For example, we could take a uniform distribution on the interior of the unit ball in $\mathbf{R}^{N}$, or let each component be an independent Gaussian random variable with mean 0 and standard deviation 1 . Our answers will hold for this entire class of distributions because our condition numbers are homogeneous (multiplying a problem by a nonzero scalar does not change its condition number).

The main justification for using a uniform distribution is that it appears to be fair: Each problem is as likely as any other. However, it does not appear to apply in many practical cases for a variety of reasons, most fundamentally because realworld problems are not uniformly distributed. A lesser reason is that any set of problems which can be represented in a computer is necessarily discrete rather than continuous. We will discuss the limitations of our choice of uniform distribution as well as alternatives at length in Section 6 below.

Finally, given this distribution, we must compute the induced probability distribution of the condition number. It turns out that all the problems we consider here have a common geometric structure which lets us compute the distributions of their condition numbers with a single analysis, which goes as follows:

(i) Certain problems of each kind are ill-posed, i.e., their condition number is infinite. These ill-posed problems form an algebraic variety within the space of all problems. For example, the singular matrices are ill-posed with respect to the problem of inversion, and they lie on the variety where the determinant, a polynomial in the matrix entries, is zero. Geometrically, varieties are possibly selfintersecting surfaces in the space of problems.

(ii) The condition number of a problem has a simple geometric interpretation: It is proportional to (or bounded by a multiple of) the reciprocal of the distance to the set of ill-posed problems. Thus, as a problem gets closer to the set of ill-posed ones, its condition number approaches infinity. In the case of matrix inversion, for example, the traditional condition number is exactly inversely proportional to the distance to the nearest singular matrix.

(iii) The last observation implies that the set of problems of condition number at least $x$ is (approximately) the set of problems within distance $c / x$ ( $c$ a constant) of the variety of ill-posed sets. Sets of this sort, sometimes called tubular neighborhoods, have been studied extensively by geometers. We will present upper bounds, lower bounds, and asymptotic values for the volumes of such sets. The asymptotic results, lower bounds, and some of the upper bounds are new. The formulae are very simple, depending only on $x$, the degree $N$ of the ambient space, the dimension of the variety, and the degree of the variety. These volume bounds in turn bound the volume of the set of problems with condition number at least $x$. Since we are assuming the problems are uniformly distributed, volume is proportional to probability.

Thus, for example, we will prove that a scaled version $\kappa(A) \equiv\|A\|_{F} \cdot\left\|A^{-1}\right\|$ of the usual condition number of a complex matrix with respect to inversion satisfies

$$
\frac{\left(1-x^{-1}\right)^{2 n^{2}-2}}{2 n^{4} x^{2}} \leq \operatorname{Prob}(\kappa(A) \geq x) \leq \frac{e^{2} n^{5}\left(1+n^{2} / x\right)^{2 n^{2}-2}}{x^{2}}
$$

and that asymptotically

$$
\operatorname{Prob}(\kappa(A) \geq x)=\frac{n\left(n^{2}-1\right)}{x^{2}}+o\left(\frac{1}{x^{2}}\right) .
$$


In other words, the probability that the condition number exceeds $x$ decreases as the square of the reciprocal of $x$. Even for moderate $x$ the upper bound exceeds the asymptotic limit by a ratio of only about $e^{2} n^{2}$. If $A$ is real we will show

$$
\frac{C(1-1 / x)^{n^{2}-1}}{x} \leq \operatorname{Prob}(\kappa(A) \geq x) \leq \sum_{k=1}^{n^{2}} 2 \cdot\left(\begin{array}{c}
n^{2} \\
k
\end{array}\right) \cdot\left(\frac{2 n}{x}\right)^{k}
$$

where $C$ is a constant proportional to the $\left(n^{2}-1\right)$-dimensional volume of the set of singular matrices inside the unit ball. Thus, for real matrices the probability that the condition number exceeds $x$ decreases as $x^{-1}$.

There are a number of open questions and conjectures concerning these volume bounds, in particular for how general a class of real varieties they apply (the case of complex varieties is simpler). We will discuss the history of this work and open problems in detail in Section 4 below.

It turns out that the reciprocal relationship between condition number and distance to the nearest ill-posed problem holds for a much wider class of problem than just matrix inversion, polynomial zerofinding and eigenvalue calculations: It is shared, at least asymptotically, by any problem whose solution is an algebraic function. For simplicity, we shall restrict ourselves to the three aforementioned problems, but our results do apply more widely, as discussed in Section 3 below and in [4].

The work was inspired by earlier work in a number of fields. Demmel [4], Gastinel [14], Hough [13], Kahan [15], Ruhe [25], Stewart [29], Wilkinson [36], [37], [38] and others have analyzed the relationship between the condition number and the distance to the nearest ill-posed problem mentioned above in (ii). Gray [8], [9], Griffiths [10], Hotelling [12], Lelong [20], Ocneanu [21], Renegar [23], Santaló [26], Smale [27], and Weyl [33] have worked on bounds of volumes of tubular neighborhoods. These volume bounds have been used by Smale [27], [28], Renegar [23] and others to analyze the efficiency of Newton's method for finding zeros of polynomials. This latter work inspired the author [3] to apply these bounds to conditioning. Ocneanu [22] and Kostlan [19] have also analyzed the statistical properties of the condition number for matrix inversion.

The rest of this paper is organized as follows. Section 2 defines notation. Section 3 discusses the relationship between conditioning and the distance to the nearest ill-posed problem. Section 4 presents the bounds on the volumes of tubular neighborhoods we shall use and states some related open problems. Section 5 computes the distributions of the condition numbers of our three problems. Section 6 discusses the limitations of assuming a uniform distribution and suggests alternatives and open problems. Section 7 contains the proofs of the theorems in Section 4 .

2. Notation. We introduce several ideas we will need from numerical analysis, algebra, and geometry. $\|x\|$ will denote the Euclidean norm of the vector $x$ as well as the induced matrix norm

$$
\|A\| \equiv \sup _{x \neq 0} \frac{\|A x\|}{\|x\|}
$$


$\|A\|_{F}$ will denote the Frobenius norm

$$
\|A\|_{F} \equiv\left(\sum_{i, j}\left|A_{i j}\right|^{2}\right)^{1 / 2}
$$

If $P$ is a set and $x$ is a point, we will let $\operatorname{dist}(x, P)$ denote the Euclidean distance from $x$ to the nearest point in $P$.

A subset $M$ of $\mathbf{R}^{N}$ is called an $n$-dimensional manifold if it is locally homeomorphic to $\mathbf{R}^{n}$. We also write $n=\operatorname{dim}(M)$. The codimension of $M$, written $\operatorname{codim}(M)$, is $N-n$. In this paper dimension will always refer to the real dimension rather than the complex dimension, which is half the real dimension.

A variety $P$ is the set of solutions of a system of polynomial equations. A variety is homogeneous if it is cone-shaped, i.e., if $x$ is in the variety so is every scalar multiple $\alpha x$. A variety is not generally a manifold since it can have singularities in the neighborhood of which it is not homeomorphic to Euclidean space. However, points $q$ with relatively open neighborhoods $U_{q} \subset P$ that are manifolds are dense in $P[17$, Theorem 4.2.4] so that the following definition makes sense: The dimension of $P$ at $p$, written $\operatorname{dim}_{p}(P)$, is

$$
\operatorname{dim}_{p}(P) \equiv \limsup _{\substack{q \rightarrow p \\ q \in U_{q} \subset P \\ U_{q} \text { a manifold }}} \operatorname{dim}\left(U_{q}\right) .
$$

We in turn define the dimension of the variety $P$ as the maximum over all $p \in P$ of $\operatorname{dim}_{p}(P)$. If $\operatorname{dim}_{p}(P)$ is constant for all $p$, we call $P$ pure dimensional. A complex variety defined by a single nonconstant polynomial is called a complex hypersurface. Complex hypersurfaces are pure dimensional with codimension 2. A real hypersurface has codimension 1 everywhere. The real variety defined by the polynomials $f_{1}, \ldots, f_{p}$ is called a complete intersection if it is pure dimensional of codimension $p$.

Now we define the degree of a purely $n$-dimensional variety $P$ in $\mathbf{R}^{N}$. Let $L^{N-n}$ be an $(N-n)$-dimensional linear manifold (plane) in $\mathbf{R}^{N}$. Since $\operatorname{dim}\left(L^{N-n}\right)+$ $\operatorname{dim}(P)=\operatorname{dim}\left(\mathbf{R}^{N}\right)=N$, we say that $L^{N-n}$ and $\mathbf{R}^{N}$ are of complementary dimension. Generically, $L^{N-n}$ and $P$ will intersect in a surface of codimension equal to the sum of their codimensions, that is $N$. In other words, their intersection will be of dimension 0 (a finite collection of points). If $P$ is a complex homogeneous variety, then for almost all planes $L^{N-n}$ this collection will contain the same number of points, and this common number is called the degree of $P$, and is written $\operatorname{deg}(P)$ (see [17, Theorem 4.6.2]). Intuitively, $\operatorname{deg}(P)$ gives the number of "leaves" of the variety $P$ that a typical plane $L^{N-n}$ will intersect. In the case of a nonhomogeneous or real variety $P, \operatorname{deg}(P)$ is defined analogously as the maximum (finite) intersection number of a plane $L^{N-n}$ and the $n$-dimensional variety $P$ in $\mathbf{R}^{N}$, although the intersection number will not generally be constant for almost all $L^{N-n}$.

This concept of degree is a generalization of the degree of a polynomial. Indeed, if $P$ is complex and defined as the solution set of a single irreducible polynomial, then the degree of the polynomial equals the degree of $P$ as defined above [17].

By $l$-volume of an $n$-dimensional manifold $M(l \geq n)$ we mean the $l$-dimensional Lebesgue measure of $M$, if it exists. Note that if $l>n$ this volume is zero. The 
notations $\operatorname{vol}(M)$ and $\operatorname{vol}_{n}(M)$ denote the $n$-volume of the $n$-dimensional manifold $M$.

3. Condition Numbers and the Distance to the Nearest Ill-Posed Problem. We claim that many classes of numerical analysis problems permit the following geometric characterization of their condition numbers:

(i) Certain problems of each class are ill-posed, i.e., their condition numbers are infinite. These problems form a variety within the space of all problems.

(ii) The condition number of a problem has a simple geometric interpretation: It is proportional to (or bounded by a multiple of) the reciprocal of the distance to the set of ill-posed problems. Thus, as a problem gets closer to the set of ill-posed ones, its condition number approaches infinity.

In this section we will cite results from the literature to prove these claims for the following three classes of problems: matrix inversion, polynomial zerofinding, and eigenvalue calculation. Afterwards we will outline why this characterization applies to many other problems as well [4].

First we need to define condition number more precisely. If $X$ is our space of problems equipped with norm $\|\cdot\|_{X}, Y$ our space of solution equipped with norm $\|\cdot\|_{Y}$, and $f: X \rightarrow Y$ is the solution map for our problem, the usual definition of the relative condition number $[24]$ is

$$
\begin{aligned}
\kappa_{\text {rel }}(f, x) & \equiv \limsup _{\delta x \rightarrow 0} \frac{\|f(x+\delta x)-f(x)\|_{Y} /\|f(x)\|_{Y}}{\|\delta x\|_{X} /\|x\|_{X}} \\
& =\frac{\|D f(x)\|_{X Y} \cdot\|x\|_{X}}{\|f(x)\|_{Y}},
\end{aligned}
$$

if the Jacobian $D f$ exists $\left(\|\cdot\|_{X Y}\right.$ is the induced norm). In many cases the essential information about the conditioning is contained in the $\|D f\|_{X Y}$ factor. We may therefore use a multiple of $\|D f\|_{X Y}$ instead of $\kappa_{\text {rel }}$ without losing essential information.

All three of our problems are homogeneous: Multiplying the problem by a scalar does not change the condition number. Therefore, the set of ill-posed problems will also be homogeneous, or cone-shaped. This permits us to normalize all our problems to have unit norm (lie on the unit sphere in either $\mathbf{R}^{N}$ or $\mathbf{C}^{N}$ ) and implies that any results on the distribution of the condition number will hold for any distribution of problems inducing the same distribution of $x /\|x\|$ on the unit sphere.

Matrix Inversion. The usual relative condition number as defined in (3.1) with the $\|\cdot\|$ norm on both the problem and solution spaces is [7]:

$$
\kappa_{\text {rel }}(A) \equiv\|A\| \cdot\left\|A^{-1}\right\| \text {. }
$$

We shall use the nearly equivalent condition number

$$
\kappa(A) \equiv\|A\|_{F} \cdot\left\|A^{-1}\right\| .
$$

These condition numbers are both homogeneous and infinite when $A$ is singular, so the set of ill-posed problems is a variety defined by the single $n$th degree homogeneous irreducible polynomial $\operatorname{det}(A)=0$, where $n=\operatorname{dim}(A)$ [32]. Denote the set of ill-posed problems by $I P$. From the last section, we see that if $A$ is complex, $I P$ 
is a complex hypersurface. If $A$ is real, it is easy to verify that $I P$ is still a real hypersurface by using the explicit parametrization provided by Gaussian elimination [3].

A theorem of Eckart and Young [5] gives the distance from a nonsingular matrix to $I P$ :

THEOREM 3.1. $\operatorname{dist}(A, I P)=\left\|A^{-1}\right\|^{-1}$.

(Gastinel [14] proved this result for an arbitrary operator norm.)

Therefore, we see that in terms of $\kappa$ we may write

$$
\text { if }\|A\|_{F}=1, \quad \text { then } \operatorname{dist}(A, I P)=1 / \kappa(A),
$$

i.e., that the distance from a normalized problem $A$ to the nearest ill-posed problem is the reciprocal of its condition number.

Polynomial Zerofinding. In this case we are interested in the sensitivity of the zeros of a polynomial to small perturbations in the coefficients. If $p(x)$ is an $n$th degree polynomial, let $\|p\|$ denote the Euclidean norm of the vector of its coefficients. If $p(z)=0$ and $\delta p$ is a small perturbation of $p$, it is easy to verify that to first order the perturbed polynomial $p+\delta p$ has a zero at $z+\delta z$ where

$$
\delta z=\frac{-\delta p(z)}{p^{\prime}(z)}
$$

implying that the relative condition number is

$$
\kappa_{\text {rel }}(p, z)=\frac{n(z) \cdot\|p\|}{\left|p^{\prime}(z)\right|}
$$

where

$$
n(z) \equiv\left|z^{-1}\right|\left(\sum_{i=0}^{n}\left|z^{2 i}\right|\right)^{1 / 2} .
$$

Note that the condition number depends both on the polynomial $p$ and the choice of zero $z$. For simplicity we will use the similar condition number

$$
\kappa(p, z) \equiv \frac{\|p\|}{\left|p^{\prime}(z)\right|}
$$

Both condition numbers are infinite when $p^{\prime}(z)=0$, i.e., when $z$ is a multiple zero. Thus we will take the set IP of ill-posed problems to be those polynomials with multiple zeros. A necessary and sufficient condition for a polynomial to have a multiple zero is that its discriminant, an irreducible homogeneous polynomial of degree $2 n-2$ in the coefficients of $p$ be zero [32]. If $p$ is complex, this implies the set of polynomials with zero discriminant is a hypersurface. If $p$ is real, this set of polynomials is still a hypersurface, as may be verified using the parametrization provided by the leading coefficient $p_{n}$ and the zeros. The discriminant may also be zero if the two leading coefficients of $p$ equal zero (corresponding to a double zero at $\infty$ ), but this set is a subvariety of double the codimension of the hypersurface in which it lies, and so forms a set of measure zero we may neglect. 
Now we need to estimate the distance from a given polynomial to one with a multiple zero. The estimate we shall use is due to Hough [13] (see also [4]) and says

THEOREM 3.2. The distance $\operatorname{dist}(p, I P)$ from the polynomial $p$ of degree at least 2 to one with a multiple zero is bounded by

$$
\operatorname{dist}(p, I P) \leq \sqrt{2} \cdot\left|p^{\prime}(z)\right|
$$

where $p(z)=0$.

In fact, this is quite a weak result gotten by estimating the smallest change in $p$ needed to make a double zero at $z$, which turns out to be a linear least-squares problem. Thus we may write

$$
\text { if }\|p\|=1, \quad \text { then } \operatorname{dist}(p, I P) \leq \frac{\sqrt{2}}{\kappa(p, z)},
$$

i.e., that the distance from a normalized problem $p$ to the nearest ill-posed problem is bounded by a multiple of the reciprocal of its condition number.

To see how much (3.3) may overestimate $\operatorname{dist}(p, I P)$, we present a lower bound. Note that by changing the argument of $p$ from $x$ to $\alpha x$ ( $\alpha$ scalar) we may make the leading coefficient $p_{n}$ larger than the other coefficients.

THEOREM $3.3([4])$. Assume that $p$ is an nth degree polynomial satisfying $\|p\|=1$ and $\left|p_{i}\right|<\left|p_{n}\right| / n$ for $i<n$. Then

$$
\operatorname{dist}(p, I P) \geq \min _{z_{i}: p\left(z_{i}\right)=0}\left(\frac{1}{n^{2}}, \frac{.0235}{n^{2} \cdot \kappa^{2}\left(p, z_{i}\right)}\right) .
$$

Thus we see that the distance to the nearest ill-posed problem is bounded below essentially by a multiple of the square of the reciprocal of the condition number. This is a general phenomenon among algebraic problems to which we return below.

Eigenvalue Calculations. We will be interested both in the sensitivity of eigenvalues and eigenvectors. More precisely, we will consider the sensitivity of the projection associated with an eigenvalue [16]. If $T$ is a matrix with simple eigenvalue $\lambda$, right eigenvector $x$ and left eigenvector $y$, the projection $P$ associated with $\lambda$ is the matrix $P=x y^{T} / y^{T} x$. The reduced resolvent associated with $\lambda$ is the matrix

$$
S \equiv \lim _{z \rightarrow \lambda}(I-P) \cdot(T-z)^{-1} .
$$

If $T$ has $n$ distinct eigenvalues $\lambda_{i}$ with projections $P_{i}$ one can write

$$
S=\sum_{\lambda_{i} \neq \lambda}\left(\lambda_{i}-\lambda\right)^{-1} P_{i}
$$

If $\delta T$ is a small perturbation of $T$, one can show [16] that to first order, $\lambda$ changes to $\lambda+\delta \lambda$ and $P$ changes to $P+\delta P$, where

$$
\delta \lambda=\operatorname{tr} P \delta T \quad \text { and } \quad \delta P=-S \delta T P-P \delta T S .
$$

It is easy to verify that $|\delta \lambda|$ can be as large as $\|P\| \cdot\|\delta T\|$, and $\|\delta P\|$ can be at least as large as $\|S\| \cdot\|P\| \cdot\|\delta T\|$ (and no more than twice as large as this). Therefore, we may take as condition numbers

$$
\kappa(T, \lambda) \equiv\|P\| \quad \text { and } \quad \kappa(T, P) \equiv\|P\| \cdot\|S\| \cdot\|T\|_{F},
$$

both of which are homogeneous. 
Both condition numbers are infinite when $\lambda$ is a multiple eigenvalue. Thus we will take the set $I P$ of ill-posed problems to be those matrices with multiple eigenvalues. We may see that $I P$ is a variety as follows. Let $p(T, \lambda)$ be the characteristic polynomial of $T$. $T$ will have multiple eigenvalues if and only if $p$ has multiple zeros, which happens if and only if the discriminant of $p$, a homogeneous polynomial of degree $n^{2}-n$ in the entries of $T$, is zero (note that $p$ is monic) [17], [32]. It is not hard to show that this polynomial is irreducible [3]. Thus we see that if $T$ is complex, IP is a hypersurface. If $T$ is real, $I P$ is still a hypersurface [1].

We now need to relate the above condition numbers of $T$ to the distance from $T$ to $I P$. A slight restatement of a theorem due to Wilkinson [36] states:

THEOREM 3.4. $\operatorname{dist}(T, I P) \leq \sqrt{2} \cdot\|T\|_{F} /\|P\|$.

Therefore, in terms of $\kappa$ we may write

$$
\text { if }\|T\|_{F}=1, \quad \text { then } \operatorname{dist}(T, I P) \leq \frac{\sqrt{2}}{\kappa(T, \lambda)} .
$$

Wilkinson's theorem provides a somewhat weak upper bound on $\operatorname{dist}(T, I P)$. The condition for $P$, on the other hand, provides [4] a lower bound on $\operatorname{dist}(T, I P)$ :

THEOREM 3.5. $\operatorname{dist}(T, I P) \geq\|T\|_{F} /(7 \cdot \kappa(T, P))$.

This result lets us write

$$
\text { if }\|T\|_{F}=1, \quad \text { then } \operatorname{dist}(T, I P) \geq \frac{1}{7 \cdot \kappa(T, P)} .
$$

For somewhat stronger results and discussion, see [4].

The phenomenon described above for matrix inversion, polynomial zerofinding and eigenvalue calculation is actually quite common in numerical analysis. It turns out all the above results can be derived from the same underlying principle, that the condition number $\kappa$ satisfies one or both of the following differential inequalities:

$$
m \cdot \kappa^{2} \leq\|D \kappa\| \leq M \cdot \kappa^{2},
$$

where $D \kappa$ is the gradient of $\kappa$. The lower bound on $\|D \kappa\|$ yields the upper bound on $\operatorname{dist}(T, I P)$,

$$
\operatorname{dist}(T, I P) \leq \frac{1}{m \cdot \kappa(T)},
$$

and the upper bound on $\|D \kappa\|$ yields the lower bound

$$
\operatorname{dist}(T, I P) \geq \frac{1}{M \cdot \kappa(T)}
$$

This phenomenon also appears in pole placement in linear control theory, Newton's method, and elsewhere [4]. In the case of algebraic functions, one can show that at least for asymptotically large condition numbers, differential inequalities of the form

$$
m \cdot \kappa^{2} \leq\|D \kappa\| \leq M \cdot \kappa^{3}
$$

hold, the new upper bound on $\|D \kappa\|$ yielding the lower bound on $\operatorname{dist}(T, I P)$,

$$
\operatorname{dist}(T, I P) \geq \frac{1}{2 M \cdot \kappa^{2}(T)}
$$

which is the source of inequality (3.4) above. 
Note also that the sets of ill-posed problems are hypersurfaces in our three examples above. Other kinds of varieties are possible as well. For example, polynomials with at most $m$ distinct zeros form a subvariety of the variety of polynomials with at least one multiple zero, and have codimension $2(n-m)$ (if complex) or $n-m$ (if real) [3]. Since $j$-tuple zeros are more sensitive than $(j-1)$-tuple zeros [35], there is a natural hierarchy of sets of ever more ill-posed problems, each one forming a subvariety of the previous set. Similar comments apply to eigenvalue calculations ( $j$-tuple eigenvalues are more sensitive than $(j-1)$-tuple eigenvalues) and rank-deficient linear least-squares problems (problems with higher rank deficiency are more sensitive than ones with lower rank deficiency). The results of the next section apply to these situations as well.

4. On Volumes of Tubes. In this section we state our main volume estimates. Proofs appear in Section 7 below:

First we consider complex varieties. The upper bourds in the following theorem are obtained by generalizing an argument of Renegar [23], who obtained the upper bound for hypersurfaces.

THEOREM 4.1. Suppose $M$ is a complex, purely $2 d$-dimensional variety in $\mathbf{C}^{N}$. Let $f(\varepsilon)$ be the fraction of the volume of the unit ball in $\mathbf{C}^{N}$ which is within distance $\varepsilon \leq 1$ of $M$. Then

$$
\begin{aligned}
f(\varepsilon) \leq & \frac{\sqrt{\pi} \Gamma(N+1 / 2)}{\Gamma(N-d+1 / 2) \Gamma(d+1 / 2)} e^{2} N^{2}(N-1)^{2 N-2 d-2} \\
& \cdot \operatorname{deg}(M) \cdot \varepsilon^{2(N-d)} \cdot(1+N \varepsilon)^{2 d} .
\end{aligned}
$$

If $M$ is a hypersurface $(d=N-1)$, then this upper bound may be improved to

$$
f(\varepsilon) \leq e^{2} N^{3} \cdot \operatorname{deg}(M) \cdot \varepsilon^{2} \cdot(1+N \varepsilon)^{2(N-1)} .
$$

If $M$ passes through the origin, it is also true that

$$
(1-\varepsilon)^{2 d} \varepsilon^{2(N-d)} \cdot \frac{\Gamma(N-d+1 / 2) \Gamma(d+1 / 2)}{\operatorname{deg}(M) \sqrt{\pi} \Gamma(N+1 / 2)} \leq f(\varepsilon) .
$$

If $M$ is a hypersurface passing through the origin, this lower bound may be improved to

$$
\frac{(1-\varepsilon)^{2 N-2} \varepsilon^{2}}{N \operatorname{deg}(M)} \leq f(\varepsilon) .
$$

Now we specialize to the case of $M$ homogeneous. In this case the upper bound (4.1) may be improved to

$$
f(\varepsilon) \leq e^{2} N^{2}(N-1)^{2 N-2 d-2} \cdot \operatorname{deg}(M) \cdot \varepsilon^{2(N-d)} \cdot(1+N \varepsilon)^{2 d} .
$$

The lower bound (4.3) may be improved to

$$
(1-\varepsilon)^{2 d} \varepsilon^{2(N-d)} \cdot \frac{\Gamma(N-d+1 / 2) \Gamma(d+1 / 2)}{\sqrt{\pi} \Gamma(N+1 / 2)} \leq f(\varepsilon) .
$$

If $M$ is also a hypersurface, the lower bound (4.4) may be further improved to

$$
\frac{(1-\varepsilon)^{2 N-2} \varepsilon^{2}}{N} \leq f(\varepsilon) \text {. }
$$


Finally, we have the following asymptotic expression for small $\varepsilon$ :

$$
f(\varepsilon)=\left(\begin{array}{l}
N \\
d
\end{array}\right) \cdot \operatorname{deg}(M) \cdot \varepsilon^{2(N-d)}+o\left(\varepsilon^{2(N-d)}\right) .
$$

Thus, we have upper bounds, lower bounds and asymptotic formulae, all of which only depend on $\varepsilon, N, d$ and $\operatorname{deg}(M)$. All our expressions are proportional to $\varepsilon^{2(N-d)}$, and for asymptotically small $\varepsilon$ differ only by factors depending on the parameters $N, \operatorname{deg}(M)$ and $d$. All these results are new, except for the upper bound (4.2) for $d=N-1$ [23].

These results can be used to give bounds for $\operatorname{Prob}(\operatorname{dist}(p, M) \leq \varepsilon)$ when $M$ is homogeneous and $p$ is uniformly distributed on the unit sphere in $\mathbf{C}^{N}$ :

THEOREM 4.2. Suppose $M$ is a complex, homogeneous, purely $2 d$-dimensional variety in $\mathbf{C}^{N}$. Let $p$ be distributed uniformly on the unit sphere centered at the origin in $\mathbf{C}^{N}$. Then for $d<N-1$

$$
\begin{gathered}
\operatorname{Prob}(\operatorname{dist}(p, M) \leq \varepsilon) \\
\leq e^{2} N^{2}(N-1)^{2 N-2 d-2} \cdot \operatorname{deg}(M) \cdot \varepsilon^{2(N-d)} \cdot(1+N \varepsilon)^{2 d}, \\
(1-\varepsilon)^{2 d} \varepsilon^{2(N-d)} \cdot \frac{\Gamma(N-d+1 / 2) \Gamma(d+1 / 2)}{2 N \sqrt{\pi} \Gamma(N+1 / 2)} \leq \operatorname{Prob}(\operatorname{dist}(p, M) \leq \varepsilon),
\end{gathered}
$$

and for asymptotically small $\varepsilon$

$$
\operatorname{Prob}(\operatorname{dist}(p, M) \leq \varepsilon)=\left(\begin{array}{c}
N-1 \\
d-1
\end{array}\right) \cdot \operatorname{deg}(M) \cdot \varepsilon^{2(N-d)}+o\left(\varepsilon^{2(N-d)}\right) .
$$

For hypersurfaces $(d=N-1)$

$$
\begin{aligned}
(1-\varepsilon)^{2 N-2} \frac{\varepsilon^{2}}{2 N^{2}} & \leq \operatorname{Prob}(\operatorname{dist}(p, M) \leq \varepsilon) \\
& \leq e^{2} N^{2} \cdot \operatorname{deg}(M) \cdot \varepsilon^{2} \cdot(1+N \varepsilon)^{2(N-1)},
\end{aligned}
$$

and for asymptotically small $\varepsilon$

$$
\operatorname{Prob}(\operatorname{dist}(p, M) \leq \varepsilon)=(N-1) \operatorname{deg}(M) \varepsilon^{2}+o\left(\varepsilon^{2}\right) .
$$

It is estimates (4.12) and (4.13) we shall apply to condition numbers in the next section.

Now we turn to real varieties. The bounds are necessarily looser, since a $d$ dimensional real variety can have an arbitrarily small volume; this is in strict contrast to complex varieties, where we can bound the volume above and below just in terms of the degree. The next theorem is due to Ocneanu [21].

THEOREM 4.3. Suppose $M$ is a real, purely d-dimensional variety in $\mathbf{R}^{N}$. Suppose further that $M$ is the complete intersection of the polynomials $g_{1}, \ldots, g_{N-d}$. Let $D \equiv \max _{i} \operatorname{deg}\left(g_{i}\right)$, and $f(\varepsilon)$ be the fraction of the volume of the unit ball in $\mathbf{R}^{N}$ which lies within distance $\varepsilon$ of $M$. Then

$$
f(\varepsilon) \leq 2(N-d) \sum_{k=N-d}^{N}\left(\begin{array}{l}
N \\
k
\end{array}\right) \cdot(2 D \varepsilon)^{k} .
$$


It appears that Ocneanu's proof can be extended to give an asymptotic formula for $f(\varepsilon)$, which we state as a

CONJECTURE. Suppose $M$ is as in Theorem 4.3. Then for asymptotically small $\varepsilon$

$$
f(\varepsilon)=\operatorname{vol}(M) \cdot \varepsilon^{N-d} \cdot \frac{N \Gamma(N / 2)}{(N-d) \pi^{d / 2} \Gamma((N-d) / 2)}+o\left(\varepsilon^{N-d}\right),
$$

where $\operatorname{vol}(M)$ is the d-dimensional volume of $M$.

Without any assumptions about complete intersection, we can compute a lower bound for $f(\varepsilon)$ :

THEOREM 4.4. Suppose $M$ is a real, purely d-dimensional variety in $\mathbf{R}^{N}$. Let $\operatorname{vol}(M[r])$ be the d-dimensional volume of the subset of $M$ within distance $r$ of the origin. Then

$$
\frac{\operatorname{vol}(M[1-\varepsilon])}{\operatorname{deg}(M)} \varepsilon^{N-d} \cdot \frac{d \Gamma(d / 2) \Gamma((d+1) / 2) \Gamma((N-d+1) / 2)}{2 \pi^{(d+1) / 2} \Gamma((N+1) / 2)} \leq f(\varepsilon) .
$$

If $M$ is homogeneous, $\operatorname{vol}(M[1-\varepsilon])$ may be replaced by $(1-\varepsilon)^{d} \operatorname{vol}(M[1])$.

Note that the ratio between the conjectured asymptotic value in (4.15) and the lower bound in (4.16) depends on $N, d$ and $\operatorname{deg}(M)$.

As before, we can translate the estimates in the last two theorems into estimates on $\operatorname{Prob}(\operatorname{dist}(p, M) \leq \varepsilon)$, where $p$ is uniformly distributed on the unit sphere:

THEOREM 4.5. Let $M$ be a real, purely d-dimensional homogeneous variety in $\mathbf{R}^{N}$. Suppose $p$ is uniformly distributed on the unit sphere in $\mathbf{R}^{N}$. Then

$$
\begin{gathered}
\frac{\operatorname{vol}(M[1])}{\operatorname{deg}(M)} \cdot(1-\varepsilon)^{d} \varepsilon^{N-d} \cdot \frac{d \Gamma(d / 2) \Gamma((d+1) / 2) \Gamma((N-d+1) / 2)}{4 N \pi^{(d+1) / 2} \Gamma((N+1) / 2)} \\
\leq \operatorname{Prob}(\operatorname{dist}(p, M) \leq \varepsilon) .
\end{gathered}
$$

If, in addition, $M$ is the complete intersection of $N-d$ polynomials each of degree at most $D$, then

$$
\operatorname{Prob}(\operatorname{dist}(p, M) \leq \varepsilon) \leq 2(N-d) \sum_{k=N-d}^{N}\left(\begin{array}{l}
N \\
k
\end{array}\right) \cdot(2 D \varepsilon)^{k} .
$$

If the conjecture (4.15) is true, then this would yield the following estimate for asymptotically small $\varepsilon$ when $M$ is a complete intersection:

$$
\begin{aligned}
& \operatorname{Prob}(\operatorname{dist}(p, M) \leq \varepsilon) \\
& \quad=\operatorname{vol}(M) \cdot \varepsilon^{N-d} \cdot \frac{d \Gamma(N / 2)}{(N-d) \pi^{d / 2} \Gamma((N-d) / 2)}+o\left(\varepsilon^{N-d}\right) .
\end{aligned}
$$

Summarizing these results for the case of a real, homogeneous hypersurface defined by a single polynomial, we have

$$
\begin{aligned}
& \frac{\operatorname{vol}(M[1])}{\operatorname{deg}(M)} \cdot(1-\varepsilon)^{N-1} \varepsilon \cdot \frac{\Gamma(N / 2)}{2 N \pi^{N / 2}} \\
& \quad \leq \operatorname{Prob}(\operatorname{dist}(p, M) \leq \varepsilon) \leq 2 \sum_{k=1}^{N}\left(\begin{array}{l}
N \\
k
\end{array}\right)(2 \operatorname{deg}(M) \varepsilon)^{k}
\end{aligned}
$$


and, for asymptotically small $\varepsilon$ (if the conjecture (4.15) is true),

$$
\operatorname{Prob}(\operatorname{dist}(p, M) \leq \varepsilon)=\operatorname{vol}(M) \cdot \varepsilon \frac{(N-1) \Gamma(N / 2)}{\pi^{N / 2}}+o(\varepsilon) .
$$

It is estimate (4.20) we will use to estimate the distribution of condition numbers of real problems.

We may explain these theorems intuitively as follows. If $M$ is a $d$-dimensional surface in $\mathbf{R}^{N}$, the dominating term in the expression for the volume of the set of points within distance $\varepsilon$ of $M$ turns out to be [33]:

(d-dimensional volume of $M$ )

$$
\cdot\left((N-d) \text {-dimensional volume of a unit ball in } \mathbf{R}^{N-d}\right) \cdot \varepsilon^{N-d} \text {. }
$$

Suppose, for example, $M$ is a straight line of length $l$ in $\mathbf{R}^{2}$. Then $d=1, N=2$ and the estimate of $(4.22)$ is $l \cdot 2 \cdot \varepsilon$, the area of a rectangle of length $l$ and width $2 \varepsilon$ centered on $M$. It turns out, even if $M$ is curved, that as long as its radius of curvature everywhere exceeds $\varepsilon$, the area of the stripe of radius $2 \varepsilon$ centered on $M$ is exactly $2 l \varepsilon$. If $M$ is a straight line of length $l$ in $\mathbf{R}^{3},(4.22)$ gives the volume $l \cdot \pi \cdot \varepsilon^{2}$ of the right circular cylinder of length $l$ and radius $\varepsilon$ centered on $M$. If $M$ is curved, this formula is still asymptotically correct for small $\varepsilon$. If $M$ is a square of side $l$ in $\mathbf{R}^{3},(4.22)$ correctly gives the volume $l^{2} \cdot 2 \cdot \varepsilon$ of the rectangular parallelepiped of thickness $2 \varepsilon$ centered on $M$. Again, bending $M$ does not change the asymptotic correctness of (4.22). In fact, if $M$ is a smooth compact manifold, for sufficiently small $\varepsilon$ the volume of the set points within distance $\varepsilon$ of $M$ is a polynomial in $\varepsilon$ with leading term given in (4.22) [33].

It remains to estimate the $d$-dimensional volume of $M$ needed in (4.22). Here we make use of the fact that $M$ is a variety, for there are formulae from integral geometry for estimating the volume of a set $M$ in $\mathbf{R}^{N}$ in terms of the number of points in $M \cap L$, where $L$ is a plane of dimension $N-d$. For varieties, this number is bounded by $\operatorname{deg}(M)$. In fact, if $M$ is a complex homogeneous purely $2 d$-dimension variety in $\mathbf{C}^{N}$, the $2 d$-volume of the part of $M$ inside the unit ball is exactly $\operatorname{deg}(M) \pi^{N} / N$ ! [30]. No such statement can be made about real varieties, so formulae like (4.3) and (4.8) cannot hold for real varieties.

Open Problems. Ocneanu's proof of Theorem 4.3 depends on the ability to express the real variety $M$ as a complete intersection. Not all varieties permit such a representation. For example, the 3 by 3 real matrices of rank at most 1 form a variety of codimension 4, but 9 polynomials (the determinants of all 2 by 2 submatrices) are needed for its definition. Is there a bound for real varieties that does not depend on the property of complete intersection? Also, Ocneanu's bound contains the factor $D^{N-d}$, which by Bezout's theorem [32] is a possibly pessimistic upper bound for $\operatorname{deg}(M)$. Is there a bound which depends only linearly on $\operatorname{deg}(M)$ ? More generally, is there an upper bound which depends linearly on $\operatorname{vol}(M[1])$ ?

All the asymptotic expressions above depend on the contribution to $f(\varepsilon)$ from small neighborhoods of the singular set of $M$ going to zero. For complex varieties, the proof of the upper bounds yields this fact. Ocneanu's proof appears to yield it as well, leading us to make conjecture (4.15).

The lower bounds for $f(\varepsilon)$ for homogeneous varieties in Theorem 4.1 are independent of $\operatorname{deg}(M)$, whereas the upper bounds are proportional to $\operatorname{deg}(M)$. By 
considering nearly overlapping hyperplanes through the origin, one can see that the lower bound cannot contain a factor of $\operatorname{deg}(M)$ and so this gap between upper and lower bounds must be present. Restricting to irreducible $M$ does not eliminate this gap (see Section 7 for discussion). However, the factor $1 / \operatorname{deg}(M)$ in the lower bounds for nonhomogeneous varieties seems unnecessary; can it be removed?

5. Computing the Distributions of Condition Numbers. In this section we apply our geometrical estimates of the last section to compute the distributions of the condition numbers discussed in Section 3.

Matrix Inversion. Applying estimates (4.12) and (4.13) to Eq. (3.2) yields the following theorem.

THEOREM 5.1. Let $A$ be a random complex $n$ by $n$ matrix distributed in such a way that $A /\|A\|_{F}$ is uniformly distributed on the unit sphere. Let $\kappa(A)=\|A\|_{F}$. $\left\|A^{-1}\right\|$. Then

$$
\frac{(1-1 / x)^{2 n^{2}-2}}{2 n^{4} x^{2}} \leq \operatorname{Prob}(\kappa(A) \geq x) \leq \frac{e^{2} n^{5}\left(1+n^{2} / x\right)^{2 n^{2}-2}}{x^{2}}
$$

and

$$
\operatorname{Prob}(\kappa(A) \geq x)=\frac{n\left(n^{2}-1\right)}{x^{2}}+o\left(\frac{1}{x^{2}}\right) .
$$

Remark. The upper bound in (5.1) exceeds the asymptotic value in (5.2) by a factor of only about $e^{2} n^{4} /\left(n^{2}-1\right)$ for sufficiently large $x$. However, even for $n=10$, $x$ must exceed about 5300 for the upper bound to drop below 1. For $n=100, x$ must exceed $2.2 \cdot 10^{7}$ for the upper bound to drop below 1. Applying estimate (4.20) to Eq. (3.2) yields

THEOREM 5.2. Let $A$ be a random real $n$ by $n$ matrix distributed in such a way that $A /\|A\|_{F}$ is uniformly distributed on the unit sphere. Let $\kappa(A)=\|A\|_{F} \cdot\left\|A^{-1}\right\|$. Then

$$
\frac{C(1-1 / x)^{n^{2}-1}}{x} \leq \operatorname{Prob}(\kappa(A) \geq x) \leq \sum_{k=1}^{n^{2}} 2\left(\begin{array}{c}
n^{2} \\
k
\end{array}\right) \cdot\left(\frac{2 n}{x}\right)^{k},
$$

where $C>0$ is a constant proportional to the volume of the variety of singular matrices inside the unit ball.

Remark. When $n=10, x$ must exceed 4900 for the upper bound in (5.3) to be less than 1. More generally, or large $n, x$ must exceed about $4.93 n^{3}$ for the upper bound to be less than 1 . One can prove this by noting that the upper bound may also be written as $2\left[(1+2 n / x)^{n^{2}}-1\right]$.

Other sets of interest are matrices of rank at most $r<n-1$. The volumes of these sets can also be estimated from above and below using Theorem 4.2, provided we can bound the degree of these varieties. Bezout's theorem [32] provides a possibly pessimistic upper bound on the degree.

Polynomial Zerofinding. Applying estimate (4.12) to inequality (3.3) yields the following theorem.

THEOREM 5.3. Let $p$ be a random complex $n$th degree polynomial distributed in such a way that $p /\|p\|_{F}$ is uniformly distributed on the unit sphere. Let 
$\kappa(p)=\max _{z}\|p\| /\left|p^{\prime}(z)\right|$, where the maximum is over all zeros of $p$. Then

$$
\operatorname{Prob}(\kappa(p) \geq x) \leq \frac{4 e(n+1)^{2}(n-1)(1+\sqrt{2}(n+1) / x)^{2 n}}{x^{2}} .
$$

Applying estimate (4.20) to inequality (3.3) yields

THEOREM 5.4. Let $p$ be a random real $n$th degree polynomial distributed in such a way that $p /\|p\|_{F}$ is uniformly distributed on the unit sphere. Let $\kappa(p)$ be as in Theorem 5.3. Then

$$
\operatorname{Prob}(\kappa(p) \geq x) \leq 2 \sum_{k=1}^{n+1}\left(\begin{array}{c}
n+1 \\
k
\end{array}\right)\left(\frac{2^{5 / 2}(n-1)}{x}\right)^{k} .
$$

Eigenvalue Calculations. Applying estimate (4.12) to inequality (3.5) yields

THEOREM 5.5. Let $A$ be a random complex $n$ by $n$ matrix distributed in such a way that $A /\|A\|_{F}$ is uniformly distributed on the unit sphere. Let $\kappa_{\lambda}(A) \equiv$ $\max _{\lambda(A)}\left\|P_{\lambda(A)}\right\|$ where the maximum is over all eigenvalues $\lambda(A)$ of $A$ and $P_{\lambda(A)}$ is the projection associated with $\lambda(A)$. Then

$$
\operatorname{Prob}\left(\kappa_{\lambda}(A) \geq x\right) \leq \frac{2 e^{2} n^{5}(n-1)\left(1+\sqrt{2} n^{2} / x\right)^{2 n^{2}-2}}{x^{2}} .
$$

Applying estimate (4.20) to inequality (3.5) yields

THEOREM 5.6. Let $A$ be a random real $n$ by $n$ matrix distributed in such a way that $A /\|A\|_{F}$ is uniformly distributed on the unit sphere. Let $\kappa_{\lambda}(A)$ be as in Theorem 5.5. Then

$$
\operatorname{Prob}\left(\kappa_{\lambda}(A) \geq x\right) \leq 2 \sum_{k=1}^{n^{2}}\left(\begin{array}{c}
n^{2} \\
k
\end{array}\right)\left(\frac{2^{3 / 2}\left(n^{2}-n\right)}{x}\right)^{k} .
$$

Applying estimate (4.12) to inequality (3.6) yields

THEOREM 5.7. Let $A$ be a random complex $n$ by $n$ matrix distributed in such a way that $A /\|A\|_{F}$ is uniformly distributed on the unit sphere. Let $\kappa_{P}(A) \equiv$ $\max _{\lambda(A)}\left\|P_{\lambda(A)}\right\| \cdot\left\|S_{\lambda(A)}\right\| \cdot\|A\|_{F}$, where the maximum is over all eigenvalues $\lambda(A)$ of $A, P_{\lambda(A)}$ is the projection associated with $\lambda(A)$, and $S_{\lambda(A)}$ is the reduced resolvent associated with $\lambda(A)$. Then

$$
\frac{(1-1 /(7 x))^{2 n^{2}-2}}{98 n^{4} x^{2}} \leq \operatorname{Prob}\left(\kappa_{P}(A) \geq x\right) .
$$

One can also prove a lower bound on $\operatorname{Prob}\left(\kappa_{P}(A) \geq x\right)$, for real matrices, which is of the form $C / x$, but $C$ is proportional to the volume of the variety of real matrices with multiple eigenvalues and lying inside the unit ball, and seems difficult to estimate.

6. Practical Applications and Limitations. In this section we show how to estimate the distribution of the error in results computed by finite precision algorithms for the problems we analyzed above. The new tool required is backward error analysis [34]; using it, we show that, except in the improbable situation that the problem to be solved is close to the set IP of ill-posed problems, a backward stable algorithm will supply an accurate answer. We analyze Gaussian elimination this way in Subsection 6.1. 
Such an analysis assumes problems are distributed uniformly as discussed in Section 1 of this paper. This is an extremely strong assumption, which is not met in many practical situations. First, real problems often have a structure which produces problems which tend to lie very close to the set $I P$ of ill-posed problems, or which in fact converge to $I P$. For example, inverse iteration to compute eigenvalues and eigenvectors involves solving a sequence of linear equations with increasingly ill-conditioned coefficient matrices. Another example is the numerical solution of differential equations; the resulting matrices are approximations of unbounded operators and become more nearly singular the finer the approximation becomes.

Second, the set of problems representable in a computer (in finite-precision arithmetic) is necessarily finite and so any distribution we put on this set will necessarily be discrete, not continuous as assumed in our previous analysis. As long as the discrete points are dense enough to model the continuum (this depends on the individual problem), the continuous model is relevant. It will turn out, however, that this discreteness ultimately leads to qualitatively different behavior of algorithms than is predicted by the continuous model. We discuss this situation further in Subsection 6.2. (This limitation does not invalidate our analysis of Gaussian elimination in finite-precision arithmetic in Subsection 6.1.)

Finally, in Subsection 6.3 we discuss how this theory might be extended to the finite-precision case and what such an extension would tell us about the design both of numerical algorithms and computer arithmetic. In particular, we show how it would tell us how many finite-precision problems we could solve as a function of the extra precision used in intermediate calculations. This information would be of use in algorithm design. Accomplishing this extension is an open problem.

6.1. A Paradigm for Analyzing the Accuracy of Finite-Precision Algorithms. The paradigm for applying the probabilistic model to the analysis of algorithms is as follows:

(1) Within the space of problems, identify the set IP of ill-posed ones and show that the closer a problem is to $I P$ the more sensitive the solution is to small changes in the problem.

(2) Show that the algorithm in question computes an accurate solution for a problem close to the one it received as input (this is known as "backward stability" [34]). Combined with the result of (1), this will show that the algorithm will compute an accurate solution to a problem as long as the problem is far enough from $I P$.

(3) Compute the probability that a random problem is close to $I P$. Using this probability distribution in conjunction with the result of (2) we can compute the probability of the algorithm computing an accurate result.

The first two steps of this paradigm are quite standard [24], [35]; only the third is new. This paradigm is best explained by applying it to matrix inversion:

(1) The set of matrices $I P$ which are ill-posed with respect to inversion are the singular matrices. As discussed in Section 3, the condition number

$$
\kappa(M)=\prod_{1} M\left\|_{F} \cdot\right\| M^{-1} \|
$$


measures how difficult the matrix $M$ is to invert, and when $\|M\|_{F}=1$ it is the reciprocal of the distance to the nearest singular matrix.

(2) Gaussian elimination with pivoting is a standard algorithm for matrix inversion and is well known to be a backward stable algorithm [34]. Backward stability means that when applying Gaussian elimination to compute the solution of the system of linear equations $M x=c$, one gets an answer $\hat{x}$ which satisfies $(M+\delta M) \hat{x}=c$, where $\delta M$ is small in norm compared to $M$. More precisely, let $X_{i}$ be the $i$ th column of the approximation to $M^{-1}$ computed using Gaussian elimination, where the arithmetic operations performed (addition, subtraction, multiplication, and division) are all rounded off to $b$ bits of precision. Then $X_{i}$ is the value of the $i$ th column of the inverse of a matrix $M+\delta M_{i}$ where $\delta M_{i}$ is small:

$$
\left\|\delta M_{i}\right\|_{F} \leq f(n) \cdot 2^{-b} \cdot\|M\|_{F}
$$

where $f(n)$ is a function only of $n$, the dimension of $M$ [34]. The magnitude of $f(n)$ depends on the pivoting strategy, and can be as large as $2^{n}$ if partial pivoting is used, although this is very rare in practice [34]. $f(n)$ is much smaller if either complete pivoting is used or if we substitute the QR algorithm for Gaussian elimination [7]. For our analysis, however, we are not interested in how big $f(n)$ is, only that inequality (6.2) holds. Inequality (6.2) can be used to bound the relative error in the computed solution [34]:

$$
\frac{\left\|X-M^{-1}\right\|_{F}}{\left\|M^{-1}\right\|_{F}} \leq \frac{\sqrt{n} \kappa(M) \cdot f(n) \cdot 2^{-b}}{1-\kappa(M) \cdot f(n) \cdot 2^{-b}} .
$$

In other words, as long as the bound (6.2) on $\left\|\delta M_{i}\right\|_{F}$ is not so large that $M+\delta M_{i}$ could be singular, i.e., as long as

$$
\operatorname{dist}(M, I P)>f(n) \cdot 2^{-b} \cdot\|M\|_{F}
$$

or, substituting from (6.1),

$$
\kappa(M)<2^{b} / f(n),
$$

then the relative error in the computed inverse $X$ is bounded, and the smaller $\kappa(M)$ is, the more accurate is the solution.

(3) Assuming $M$ is complex, we can apply Theorem 5.1 (which gives the probability distribution of the condition number) to estimate the probability that a random matrix can be inverted accurately:

$$
\operatorname{Prob}\left(\frac{\left\|X-M^{-1}\right\|_{F}}{\left\|M^{-1}\right\|_{F}} \leq \varepsilon\right) \geq \operatorname{Prob}\left(\frac{\sqrt{n} \kappa(M) \cdot f(n) \cdot 2^{-b}}{1-\kappa(M) \cdot f(n) \cdot 2^{-b}} \leq \varepsilon\right),
$$

which, after some rearrangement (and assuming $\varepsilon<1$ ) equals

$$
\begin{aligned}
\operatorname{Prob} & \left(\kappa(M) \leq \frac{\varepsilon}{f(n) \cdot(\sqrt{n}+\varepsilon) 2^{-b}}\right) \\
& \geq 1-\left(e^{2} n^{5}\left(1+n^{2} f(n)(\sqrt{n}+\varepsilon) \cdot\left(2^{-b} / \varepsilon\right)\right)^{2 n^{2}-2} f^{2}(n)(\sqrt{n}+\varepsilon)^{2}\right) \cdot\left(\frac{2^{-b}}{\varepsilon}\right)^{2} \\
& \equiv 1-g(n, \varepsilon, b) \cdot\left(2^{-b} / \varepsilon\right)^{2} .
\end{aligned}
$$

The $g(n, \varepsilon, b)$ factor depends only weakly on $\varepsilon$ and $b$; the interesting factor is $\left(2^{-b} / \varepsilon\right)^{2}$. This inequality implies that as we compute with higher and higher precision ( $b$ increases), the probability of getting a computed answer with accuracy 
$\varepsilon$ goes to 1 at least as fast as $1-O\left(4^{-b}\right)$. Note that the inequality only makes sense for $2^{-b} / \varepsilon$ small, that is, if the error $2^{-b}$ in the arithmetic is smaller than the error $\varepsilon$ demanded of the answer. This restriction makes sense numerically, since we cannot expect more precision than we compute with. The restriction also implies that the finite-precision numbers are sufficiently dense to approximate the continuum, since the radius $r$ of the neighborhood around $I P, r=f(n)(\sqrt{n}+\varepsilon) 2^{-b} / \varepsilon$, is much larger than the distance between adjacent finite-precision points $2^{-b}$. This situation is depicted in Figure 1 and discussed in the next section.

We may use the same kind of paradigm as discussed so far to analyze the speed of convergence of an algorithm rather than its accuracy. In this case the paradigm is

$\left(1^{\prime}\right)$ Identify the ill-posed problems $I P$.

$\left(2^{\prime}\right)$ Show that the closer a problem is to $I P$, the more slowly the algorithm converges.

$\left(3^{\prime}\right)$ Compute the probability that a random problem is close to $I P$. Combined with $\left(2^{\prime}\right)$ this yields the probability distribution of the speed of convergence.

This approach has been used by Smale [27] and Renegar [23] in their average speed analyses of Newton's method for finding zeros of polynomials.

6.2. Limitations of the Probabilistic Model. In this section we discuss limitations to the applicability of our model. As mentioned before, the model does not apply in situations where the problems tend to be clustered about the ill-posed problems. One might even assert that most problems have this character, or at least constitute the majority of interesting problems numerical analysts encounter. One such example is iteration for computing the eigenvalues and eigenvectors of a matrix:

$$
\begin{aligned}
x_{i+1} & =\left(A-\lambda_{i}\right)^{-1} x_{i}, \\
\lambda_{i+1} & =\left(A x_{i+1}\right)^{j} / x_{i+1}^{j}, \quad \text { where }\left|x_{i+1}^{j}\right|=\max _{k}\left|x_{i+1}^{k}\right| .
\end{aligned}
$$

If $\lambda_{i}$ is a good approximation to the simple eigenvalue $\lambda$, and $x_{i}$ approximates the corresponding eigenvector $x$, then $\lambda_{i+1}$ and $x_{i+1}$ will be even better approximations to $\lambda$ and $x$. As $\lambda_{i}$ approaches $\lambda$, the matrices $A-\lambda_{i}$ become increasingly illconditioned. Thus, the set of matrices $\left\{A-\lambda_{i}\right\}$ being (conceptually) inverted (actually, one solves $\left(A-\lambda_{i}\right) x_{i+1}=x_{i}$ directly) converges to the set $I P$ of illposed problems, and so is far from uniformly distributed. This invalidates the assumption of the model, even in exact arithmetic. In finite-precision arithmetic, inverse iteration works very well, even though naive backward error analysis as in Subsection 6.1 might lead us to expect total loss of precision. This is because the rounding errors committed while solving $\left(A-\lambda_{i}\right) x_{i+1}=x_{i}$ provably conspire to produce an error lying almost certainly in the direction of the desired eigenvector [7].

Another area where matrices tend to cluster around the singular ones is the solution of differential equations. In this case the matrices encountered are generally approximations to unbounded operators, and so become more nearly singular the finer the approximation. For example, the usual centered difference approximation to the second derivative on the interval $[0,1]$ with mesh spacing $h$ yields a matrix with condition number on the order of $h^{-2}$. 


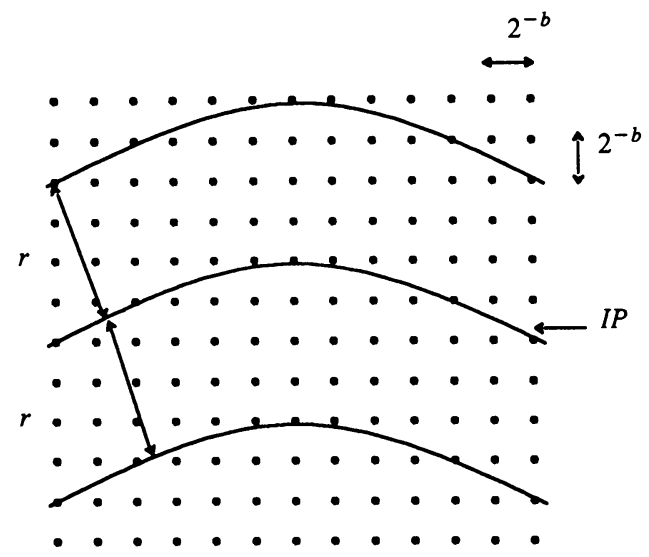

FIGURE 1

An $r>2^{-b}$ neighborhood of IP

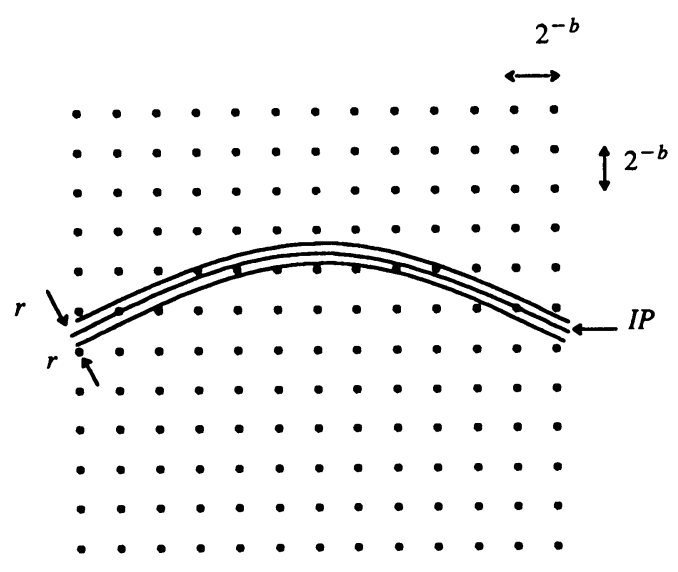

FIGURE 2

An $r<2^{-b}$ neighborhood of IP

The second way in which the model breaks down depends on the ultimate discreteness of the finite-precision numbers which can be represented in a computer. The natural version of a "uniform distribution" in this case is simply counting measure. The continuous model is a good approximation to counting measure only as long as the finite-precision numbers are dense enough to resemble the continuum. In Figure 1, for example, the area of the set of points within distance $r$ of the curve $I P$ is a good approximation to the number of dots (finite-precision points) within distance $r$ of $I P$ (scaled appropriately). This is true because the radius $r$ of the neighborhood of $I P$ is large compared to the spacing $2^{-b}$ between dots. When $r<2^{-b}$ on the other hand, as in Figure 2, the area of the set of points within distance $r$ of $I P$ is not necessarily a good approximation of the number of dots within 
$r$ of $I P$. For example, if $I P$ were a straight line passing exactly half way between two rows of dots, there would be no dots within distance $2^{-b-1}$ of $I P$. If, on the other hand, $I P$ were a straight line running along a row of dots, there would be a constant nonzero number of dots within distance $\eta$ of $I P$ for all $\eta<2^{-b}$. Thus, when the radius of the neighborhood of $I P$ gets smaller than the interdot distance $2^{-b}$, the model breaks down.

Specifically, let us consider matrix inversion. In the continuous model, the exactly singular matrices form a set of measure zero, so the chance of a random problem being singular is zero. Also, there are nonsingular matrices arbitrarily close to the set of singular ones, and so of unbounded condition number. Consider now the finite (but large) set of matrices which can be represented in a computer using finite-precision arithmetic. Some fraction of this finite set are exactly singular, so in choosing one member of this finite set at random (using counting measure), there is a nonzero probability of getting an exactly singular matrix. Furthermore, the remaining nonsingular matrices have condition numbers bounded by some finite value $K$. Thus, instead of $\operatorname{Prob}(\kappa(A) \geq x)$ decreasing monotonically to 0 as $x$ increases, as in the continuous case, $\operatorname{Prob}(\kappa(A) \geq x)$ becomes constant and nonzero for $x>K$. This is clearly significantly different behavior. It does not, however, invalidate the analysis of Gaussian elimination in the last section, because we assumed $2^{-b}<r$, i.e., the situation in Figure 1 .

In the next section we discuss what we could do if we could compute $\operatorname{Prob}(\kappa(A) \geq$ $x$ ) in the discrete case for all $x$, in particular for $x$ too large for the continuous approximation to apply.

6.3. How to Use the Discrete Distribution of Points Within Distance $\varepsilon$ of a Variety. Before proceeding, we need to say what probability measure we are going to put on the discrete set of finite-precision points. The last section showed that no single distribution is good for all applications, but a uniform distribution remains a neutral and interesting choice. So far, we have been implicitly using fixed-point numbers, in which case assigning equal probability to each point (counting measure) gives a uniform distribution. For floating-point numbers, however, this is no longer appropriate since the floating-point numbers are not evenly distributed on the number line. Since floating-point numbers are much closer together near the origin than far away from it (the distance between adjacent numbers is approximately a constant times the number), counting measure would assign much more probability to equal length intervals near the origin than far away from it. A simple way to adjust for this nonuniform spacing is to assign to each point $M$ a probability proportional to the volume of the small parallelepiped of points which round to $M$ (i.e., the parallelepiped centered at $M$ with sides equal in length to the distance between adjacent finite-precision points). In the case of fixed-point arithmetic, this just reproduces counting measure, whereas with floating-point arithmetic, points near 0 have smaller probability than larger points, and intervals of equal length have approximately equal probabilities. Actually, the question of the distribution of the digits of a floating-point number has a large literature [2], [11], [18], but the discussion in this section does not depend strongly on the actual distribution of digits chosen. 
This discussion does assume that the finite-precision input is known exactly, i.e., that there is no error inherited from previous computations or from measurement errors. In general there will be such errors, and they will almost always be at least a few units in the last place of the input problem. In other words, there already is a ball of uncertainty around the input problem with a radius equal to a small multiple of the interpoint distance $2^{-b_{0}}\left(b_{0}\right.$ is the number of bits to which the input is stored). Therefore, it may make no sense to use higher precision to accurately solve problems lying very close to $I P$ when the inherited input error is so large that the true answer is inherently very uncertain. In such situations, programmers sometimes shrug and settle for the backward stability provided by the algorithm, even if the delivered solution is entirely wrong, because the act of solution has scarcely worsened the uncertainty inherited from the data, and the programmer declines to be held responsible for the uncertainty inherent in the data. Nevertheless, getting an accurate answer for as many inputs as possible is a worthwhile goal, so we will not concern ourselves with possible errors made in creating the input matrices.

We claim that knowing the probability distribution of the distance of a random finite-precision problem to the set IP of ill-posed problems would tell us how many finite-precision problems we could solve as a function of the extra precision used in intermediate calculations. As mentioned before, programmers often resort to extra precision arithmetic to get more accurate solutions to problems which are given only to single precision. This extra precision has a cost (in speed and memory) dependent on the number of digits carried, so programmers usually avoid extra precision unless persuaded otherwise by bad experiences, an error analysis, or paranoia. Therefore, an accurate estimate of how many problems can be solved as a function of the extra precision would help programmers decide how much to use.

How does knowledge of this probability distribution tell us how much extra precision to use? The paradigm in Subsection 6.1 tells us how. Consider matrix inversion. Formula (6.3) tells us that using fixed-point arithmetic of accuracy $2^{-b}$ permits us to compute inverses of matrices to within accuracy $\varepsilon$ as long as their condition numbers are less than $\varepsilon /\left(f(n)(\sqrt{n}+\varepsilon) 2^{-b}\right)$. Suppose we choose our problems at random from the set of matrices with $b_{0}$-bit entries, and let $\operatorname{Prob}_{b_{0}}(\kappa(M) \geq x)$ be the discrete distribution function of the condition number. Then

$$
N_{b_{0}}(b) \equiv 1-\operatorname{Prob}_{b_{0}}\left(\kappa(M) \geq \frac{\varepsilon}{f(n)(\sqrt{n}+\varepsilon) 2^{-b}}\right)
$$

bounds from below the fraction of $b_{0}$-bit matrices we can invert with accuracy $\varepsilon$ as a function of the number of bits $b \geq b_{0}$ carried in the calculation. By examining $N_{b_{0}}(b)$ as a function of $b$, one can decide exactly how much improvement one gets for each additional bit of precision $b$. For example, we know from previous discussion that there is a $\bar{b}$ such that, when $b \geq \bar{b}, N_{b_{0}}(b)$ is constant and nonzero. Therefore, it clearly does not pay to increase $b$ beyond $\bar{b}$.

We close with an example of the discrete distribution $\operatorname{Prob}_{b_{0}}(\kappa(M) \geq x)$. Consider the rather simple problem of inverting real 2 by 2 matrices. This problem is small enough that we can exhaustively compute $\operatorname{Prob}_{b_{0}}(\kappa(M) \geq x)$ for low-precision arithmetic. We have done this for $b_{0}=3,4,5,6$ and 7 (all numbers lay between 0 
and 1 in absolute value, and each fixed-point matrix was assigned the same probability). Let $P(r)=\operatorname{Prob}_{b_{0}}(\kappa(M) \geq 1 / r)$. We recall that in the continuous case (Theorem 5.2) $P(r)$ would be approximately a linear function of $r$. For all values of $b_{0}$ tested, we observed approximately the behavior of $P(r)$ shown in Figure 3. Surprisingly, we observed linear dependence of $P(r)$ on $r$ not only for $r$ larger than $2^{-b_{0}}$ (corresponding to Figure 1), but for $r$ quite a bit smaller than $2^{-b_{0}}$ (Figure $2)$. The fraction of problems within $2^{-b_{0}}$ of a singular matrix was about $2^{1-b_{0}}$. This linear behavior of $P(r)$ continued until $r$ reached approximately $2^{-2 b_{0}}$, and there the graph of the distribution became horizontal and remained so all the way to the origin, intersecting the vertical axis at about $2^{2-2 b_{0}}$. This means that all matrices closer to $I P$ than approximately $2^{-2 b_{0}}$ were exactly singular. The fraction of matrices which were exactly singular was $2^{2-2 b_{0}}$.

What does this tell us about the use of extra precision? Basically, as long as the distribution function $P(r)$ remains linear, it says that for every extra bit of intermediate precision, we can solve half the problems we could not solve before. This regime continues until we reach double precision, at which point the only problems we cannot solve are exactly singular. Indeed, since

$$
\left[\begin{array}{ll}
a & c \\
b & d
\end{array}\right]^{-1}=(a d-b c)^{-1}\left[\begin{array}{cc}
d & -c \\
-b & a
\end{array}\right]
$$

we can clearly compute the inverse accurately if we can compute the determinant $a d-b c$ accurately. Since $a, b, c$ and $d$ are given to single precision, double precision clearly suffices to compute $a d-b c$ exactly.

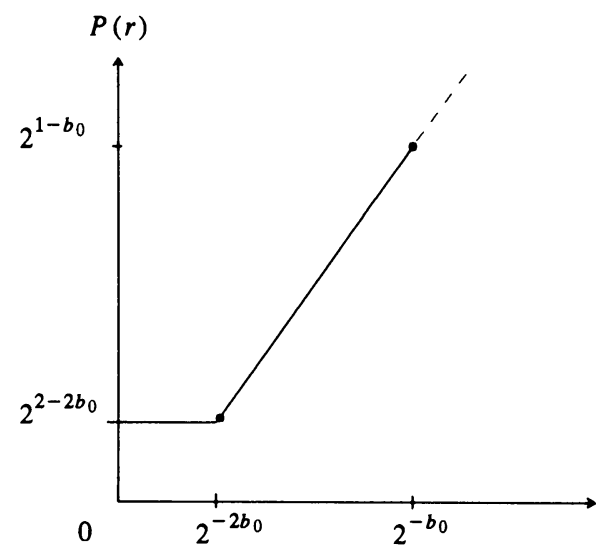

FiguRE 3

Observed probability distribution of the distance $r$ to the nearest singular matrix.

What if the discrete distribution function were similar for matrices of higher dimensions, that is, linear for a while and then suddenly horizontal when all worseconditioned matrices were exactly singular? It would again tell us that for a while, every extra bit of intermediate precision would let us solve half the problems we 
could not solve before. Eventually, after enough extra bits (and for inverting fixedprecision $n$ by $n$ matrices, this clearly occurs no later than reaching $n$-tuple precision), all finite-precision matrices which are not exactly singular could be inverted, and more precision would contribute nothing. Thus a programmer (or system designer) could choose the number of bits $b$ with which to compute in order to guarantee that the fraction of unsolvable problems is sufficiently close to its minimum. Of course, exhaustive evaluation of the distribution function is not reasonable for large problems, and estimating the distribution function becomes an interesting open question of number theory.

7. Proofs of Volume Estimates. In this section we present the proofs of the volume estimates of Section 4. In addition to the notation of Section 2, we will use

$$
O_{n}=\frac{2 \pi^{(n+1) / 2}}{\Gamma((n+1) / 2)} \quad \text { and } \quad \theta_{n}=\frac{O_{n-1}}{n}=\frac{2 \pi^{n / 2}}{n \Gamma(n / 2)} .
$$

$O_{n}$ is the surface area of the $n$-dimensional unit sphere and $\theta_{n}$ is the volume of the $n$-dimensional unit ball [26]. $B(p, r)$ is the open ball of radius $r$ centered at $p$, and $B(r)$ is centered at the origin. If $M$ is any set, $M[r]=M \cap \bar{B}(r)$. If $M$ is a variety, $N S(M)$ will denote the nonsingular part of $M$ (a manifold) and $S(M)$ the remaining singular part (a lower-dimensional subvariety). $\operatorname{vol}(M)$ or $\operatorname{vol}_{\operatorname{dim}(M)}(M)$ will denote the $\operatorname{dim}(M)$-dimensional measure of $N S(M) . T(M, \varepsilon)$ is the set of points inside $\bar{B}(1)$ within distance $\varepsilon$ of $M$ :

$$
T(M, \varepsilon)=\{z:\|z\| \leq 1, \operatorname{dist}(z, M) \leq \varepsilon\} .
$$

If $M \subseteq \mathbf{R}^{N}, f(M, \varepsilon)=\operatorname{vol}_{N}(T(M, \varepsilon)) / \theta_{N}$, the fraction of the unit ball within $\varepsilon$ of $M$. \# $(S)$ will denote the cardinality of the discrete set $S$.

We will need the following estimates on the volumes of varieties inside balls.

LEMMA 7.1 ([31]). Let $M$ be a purely $2 d$-dimensional homogeneous complex variety in $\mathbf{C}^{N}$. Then $\operatorname{vol}_{2 d}(M[r])=\operatorname{deg}(M) \cdot \theta_{2 d} \cdot r^{2 d}$.

LEMMA 7.2 ([30, Theorem B]). Let $M$ be a purely $2 d$-dimensional complex variety containing the origin. Then $\operatorname{vol}_{2 d}(M[r]) \geq \theta_{2 d} \cdot r^{2 d}$.

LEMMA 7.3 ([23, Proposition 5.3]). Let $M$ be a complex hypersurface in $\mathbf{C}^{N}$. Then $\operatorname{vol}_{2 N-2}(M[r]) \leq \operatorname{deg}(M) \cdot N \cdot \theta_{2 N-2} \cdot r^{2 N-2}$.

LEMMA 7.4. Let $M$ be a purely d-dimensional real variety in $\mathbf{R}^{N}$. Then

$$
\operatorname{vol}_{d}(M[r]) \leq \operatorname{deg}(M) \cdot \frac{O_{N-d} \cdot O_{d}}{2 \cdot O_{N}} \cdot \theta_{d} \cdot r^{d}
$$

Proof. That $\operatorname{vol}_{d}(M[r])$ is finite follows from [6, Section 3.4.10]. Let $L_{N-d}$ denote an $(N-d)$-dimensional plane in $\mathbf{R}^{N} \cdot d L_{N-d}$ will denote the kinematic density on this set of planes [26, Chapter 12]. From [26, Eq. 12.38] we may write $d L_{N-d}=d \sigma_{d} \wedge d L_{d[0]}$, where $d L_{d[0]}$ is the kinematic density on $d$-planes through the origin and $d \sigma_{d}$ is the volume element on $L_{d[0]}$. This corresponds to parametrizing a plane $L_{N-d}$ by the perpendicular plane through the origin $L_{d[0]}$ which it intersects, and where it intersects it. From [26, Eq. 14.70] we may write

$$
\int_{M[r] \cap L_{N-d} \neq \varnothing} \#\left(M[r] \cap L_{N-d}\right) d L_{N-d}=\frac{O_{N} \cdots O_{d+1}}{O_{N-d} \cdots O_{1}} \operatorname{vol}_{d}(M[r])
$$


or

$$
\begin{aligned}
\operatorname{vol}_{d}(M[r]) & =\frac{O_{N-d} \cdots O_{1}}{O_{N} \cdots O_{d+1}} \int \#\left(M[r] \cap L_{N-d}\right) d L_{N-d} \\
& \leq \frac{O_{N-d} \cdots O_{1}}{O_{N} \cdots O_{d+1}} \cdot \operatorname{deg}(M) \cdot \int_{\|x\| \leq r} d \sigma_{d} \wedge d L_{d[0]}
\end{aligned}
$$

where $x$ is the intersection point of $L_{N-d}$ and $L_{d[0]}$. In other words, $M[r]$ and $L_{N-d}$ can intersect only if $L_{N-d}$ passes within distance $r$ of the origin. The last displayed expression in turn equals

$$
\begin{aligned}
& \frac{O_{N-d} \cdots O_{1}}{O_{N} \cdots O_{d+1}} \cdot \operatorname{deg}(M) \cdot \int_{\|x\| \leq r} d \sigma_{d} \cdot \int d L_{d[0]} \\
& \quad=\frac{O_{N-d} \cdots O_{1}}{O_{N} \cdots O_{d+1}} \cdot \operatorname{deg}(M) \cdot r^{d} \cdot \theta_{d} \cdot \frac{O_{N-1} \cdots O_{N-d}}{O_{d-1} \cdots O_{0}} \\
& \quad=\frac{O_{N-d} \cdot O_{d}}{O_{0} \cdot O_{N}} \cdot \operatorname{deg}(M) \cdot \theta_{d} \cdot r^{d}
\end{aligned}
$$

where we have used [26, Eq. 12.35].

By considering a purely $2 d$-dimensional complex variety in $\mathbf{C}^{N}$ as a real variety in $\mathbf{R}^{2 N}$, the last lemma implies

COROllaRY 7.5. Let $M$ be a purely $2 d$-dimensional complex variety in $\mathbf{C}^{N}$. Then

$$
\operatorname{vol}_{2 d}(M[r]) \leq \operatorname{deg}(M) \cdot \frac{O_{2 N-2 d} \cdot O_{2 d}}{2 \cdot O_{2 N}} \cdot \theta_{2 d} \cdot r^{2 d}
$$

Remark. In the case of hypersurfaces $(d=N-1)$, Corollary 7.5 yields the bound $\operatorname{deg}(M) \cdot(2 N-1) \cdot \theta_{2 N-2} \cdot r^{2 N-2}$, which is about a factor of 2 weaker than Lemma 7.3. This is because the proof of Lemma 7.4 takes no advantage of the complex analytic structure of $M$. One might be able to improve Corollary 7.5 by taking advantage of this structure.

LEMMA 7.6. Let $M$ be a purely $2 d$-dimensional complex variety in $\mathbf{C}^{N}$. Let $s=N$ if $M$ is a hypersurface $(d=N-1)$ and $s=O_{2 N-2 d} O_{2 d} /\left(2 O_{2 N}\right)$ otherwise. Then

$$
\operatorname{vol}_{2 N}(T(M, \varepsilon)) \geq \frac{\operatorname{vol}_{2 d}(M[1-\varepsilon])}{\operatorname{deg}(M) \cdot s \cdot \theta_{2 d}} \cdot \theta_{2 N} \cdot \varepsilon^{2(N-d)} .
$$

Proof. Following [26, Chapter 15], we will let $d K$ be the kinematic density for the group of Euclidean motions in $\mathbf{C}^{N} \equiv \mathbf{R}^{2 N}$. $d K$ may be written $d A \wedge d b$, where $d A$ is a density on the special orthogonal group $S O(2 N), d b$ is Lebesgue measure on $\mathbf{R}^{2 N}$, and $K(s)=A s+b$. From [26, Eq. 15.20] we have

$$
\begin{aligned}
& \int_{M[1-\varepsilon] \cap K(B(\varepsilon)) \neq \varnothing} \operatorname{vol}_{2 d}(M[1-\varepsilon] \cap K(B(\varepsilon))) d K \\
& =O_{2 N-1} \cdots O_{1} \cdot \operatorname{vol}_{2 d}(M[1-\varepsilon]) \cdot \operatorname{vol}_{2 N}(B(\varepsilon)),
\end{aligned}
$$

where $K(B(\varepsilon))$ is the Euclidean motion $K$ applied to $B(\varepsilon)$. (Actually, Santaló only proved this theorem, as well as others of his we shall use, for closed manifolds. The same results for rectifiable surfaces (including varieties) are due to Federer [6, Theorem 3.2.48 and Section 3.4.10]. We will continue to use Santaló's formulation of the result because it is more convenient.) Since $\operatorname{vol}_{2 N}(B(\varepsilon))=\theta_{2 N} \varepsilon^{2 N}$ and 
$\operatorname{vol}_{2 d}(M[1-\varepsilon] \cap K(B(\varepsilon))) \leq \operatorname{deg}(M) \cdot s \cdot \theta_{2 d} \cdot \varepsilon^{2 d}$ by Lemma 7.3 and Corollary 7.5 , we have

$$
\begin{array}{r}
\operatorname{deg}(M) \cdot s \cdot \theta_{2 d} \cdot \varepsilon^{2 d} \cdot \int_{M[1-\varepsilon] \cap K(B(\varepsilon)) \neq \varnothing} d K \\
\quad \geq O_{2 N-1} \cdots O_{1} \cdot \operatorname{vol}_{2 d}(M[1-\varepsilon]) \cdot \theta_{2 N} \cdot \varepsilon^{2 N} .
\end{array}
$$

Since $B(\varepsilon)$ is a ball, $K(B(\varepsilon))=B(b, \varepsilon)$, so

$$
\begin{gathered}
\int_{M[1-\varepsilon] \cap K(B(\varepsilon)) \neq \varnothing} d K=\int_{M[1-\varepsilon] \cap B(b, \varepsilon) \neq \varnothing} d A \wedge d b \\
=\int_{M[1-\varepsilon] \cap B(b, \varepsilon) \neq \varnothing} d b \int d A=O_{2 N-1} \cdots O_{1} \cdot \int_{M[1-\varepsilon] \cap B(b, \varepsilon) \neq \varnothing} d b
\end{gathered}
$$

where we have used [26, Eq. 12.11]. Now $\int_{M[1-\varepsilon] \cap B(b, \varepsilon) \neq \varnothing} d b$ is the volume of the set of points within distance $\varepsilon$ of $M[1-\varepsilon]$, which is included in $T(M, \varepsilon)$. Therefore,

$$
\int_{M[1-\varepsilon] \cap B(b, \varepsilon) \neq \varnothing} d b \leq \operatorname{vol}_{2 N}(T(M, \varepsilon)) .
$$

Combining (7.2), (7.3) and (7.4) yields the result.

THEOREM 7.7. Let $M$ be a purely $2 d$-dimensional complex variety in $\mathbf{C}^{N}$ containing the origin. If $d<N-1$, then

$$
\frac{(1-\varepsilon)^{2 d} \varepsilon^{2(N-d)}}{\operatorname{deg}(M)} \cdot \frac{2 O_{2 N}}{O_{2 N-2 d} O_{2 d}} \leq f(M, \varepsilon) .
$$

If $d=N-1$ ( $M$ a hypersurface $)$, then

$$
\frac{(1-\varepsilon)^{2 N-2} \varepsilon^{2}}{N \cdot \operatorname{deg}(M)} \leq f(M, \varepsilon) .
$$

If $M$ is homogeneous, the lower bounds in both (7.5) and (7.6) may be increased by the factor $\operatorname{deg}(M)$.

Proof. Both (7.5) and (7.6) follow from dividing (7.1) by $\theta_{2 N}$ and using the estimate $\operatorname{vol}_{2 d}(M[1-\varepsilon]) \geq \theta_{2 d}(1-\varepsilon)^{2 d}$ from Lemma 7.2. For homogeneous $M$, use Lemma 7.1 instead.

The proof of Lemma 7.6 depended on the complex analyticity of $M$ only in the estimate

$$
\operatorname{vol}_{2 d}(M[1-\varepsilon] \cap K(B(\varepsilon))) \leq \operatorname{deg}(M) \cdot s \cdot \theta_{2 d} \cdot \varepsilon^{2 d},
$$

based on Lemma 7.3 and Corollary 7.5. If $M$ is a $d$-dimensional real variety in $\mathbf{R}^{N}$, we can use Lemma 7.4 to instead estimate

$$
\operatorname{vol}_{d}(M[1-\varepsilon] \cap K(B(\varepsilon))) \leq \operatorname{deg}(M) \cdot \frac{O_{N-d} \cdot O_{d}}{2 \cdot O_{N}} \cdot \theta_{d} \cdot \varepsilon^{d}
$$

Using this bound in the proof of Lemma 7.6 yields

LEMMA 7.8. Let $M$ be a purely d-dimensional real variety in $\mathbf{R}^{N}$. Then

$$
\operatorname{vol}_{N}(T(M, \varepsilon)) \geq \frac{\operatorname{vol}_{d}(M[1-\varepsilon])}{\operatorname{deg}(M) \cdot \theta_{d}} \cdot \frac{2 O_{N}}{O_{N-d} \cdot O_{d}} \cdot \theta_{N} \cdot \varepsilon^{N-d}
$$


This immediately yields

THEOREM 7.9. Let $M$ be a purely d-dimensional real variety in $\mathbf{R}^{N}$. Then

$$
\frac{\operatorname{vol}_{d}(M[1-\varepsilon])}{\operatorname{deg}(M) \cdot \theta_{d}} \frac{2 O_{N}}{O_{N-d} O_{d}} \cdot \varepsilon^{N-d} \leq f(M, \varepsilon) .
$$

If $M$ is homogeneous, then

$$
\frac{(1-\varepsilon)^{d} \operatorname{vol}_{d}(M[1])}{\operatorname{deg}(M) \cdot \theta_{d}} \cdot \frac{2 O_{N}}{O_{N-d} O_{d}} \cdot \varepsilon^{N-d} \leq f(M, \varepsilon) .
$$

Proof. (7.9) follows by dividing (7.7) by $\theta_{N}$. When $M$ is homogeneous, $\operatorname{vol}_{d}(M[r])$ $=r^{d} \operatorname{vol}_{d}(M[1])$.

Remark. This theorem has the unhappy property that when $M$ is concentrated near the boundary of $B(1)$, the lower bound degenerates to 0 since

$$
\operatorname{vol}_{d}(M[1-\varepsilon])=0 \text {. }
$$

Consideration of several examples of this kind leads us to conjecture that $\operatorname{vol}_{d}(M[1-\varepsilon])$ may be replaced by

$$
\operatorname{vol}_{d}(M[1]) \frac{1-(1-\varepsilon)^{N}}{(1+\varepsilon)^{N}-(1-\varepsilon)^{N}} \geq \frac{\operatorname{vol}_{d}(M[1])}{2^{N}},
$$

where $\varepsilon \leq 1$.

This completes all our lower bounds. The lower bounds in Theorems 4.1 and 4.4 follow from substituting for $O_{i}$ and $\theta_{j}$ in Theorems 7.7 and 7.9.

We now turn to upper bounds. The following theorem generalizes a technique used in [23, Proposition 5.4].

LEMMA 7.10. Let $M$ be a purely 2 d-dimensional complex variety in $\mathbf{C}^{N}$. Let

$$
s=\left\{\begin{array}{l}
1 \quad \text { if } M \text { is homogeneous, } \\
N \text { if } M \text { is a nonhomogeneous hypersurface }(d=N-1), \\
O_{2 N-2 d} O_{2 d} /\left(2 O_{2 N}\right) \text { otherwise. }
\end{array}\right.
$$

Then

(7.11) $\operatorname{vol}_{2 N}(T(M, \varepsilon)) \leq e^{2} N^{2}(N-1)^{2(N-d-1)} \cdot s \cdot \operatorname{deg}(M) \cdot \theta_{2 N} \cdot(1+N \varepsilon)^{2 d} \cdot \varepsilon^{2(N-d)}$.

Proof. Let $d K$ be the kinematic density for the group of Euclidean motions as in the proof of Lemma 7.6. Then from [26, Eq. 15.20] we have

$$
\begin{aligned}
& \int \operatorname{vol}_{2 d}(M[1+N \varepsilon] \cap K(B(N \varepsilon))) d K \\
& \quad=O_{2 N-1} \cdots O_{1} \cdot \operatorname{vol}_{2 N}(B(N \varepsilon)) \cdot \operatorname{vol}_{2 d}(M[1+N \varepsilon])
\end{aligned}
$$

where the integral is over all motions $K$ such that $M[1+N \varepsilon] \cap K(B(N \varepsilon)) \neq \varnothing$. Since

$\operatorname{vol}_{2 N}(B(N \varepsilon))=\theta_{2 N}(N \varepsilon)^{2 N} \quad$ and $\quad \operatorname{vol}_{2 d}(M[1+N \varepsilon]) \leq \operatorname{deg}(M) \cdot \theta_{2 d} \cdot(1+N \varepsilon)^{2 d} \cdot s$

by Lemmas 7.1, 7.3 and 7.4, we have

$$
\begin{aligned}
& \int \operatorname{vol}_{2 d}(M[1+N \varepsilon] \cap K(B(N \varepsilon))) d K \\
& \quad \leq O_{2 N-1} \cdots O_{1} \cdot \theta_{2 N} N^{2 N} \varepsilon^{2 N} \cdot \operatorname{deg}(M) \cdot \theta_{2 d} \cdot(1+N \varepsilon)^{2 d} \cdot s
\end{aligned}
$$


Recall that $K(s)=A s+b$, where $A \in S O(2 N)$, and $d K=d A \wedge d b$. Thus $K(B(N \varepsilon))=B(b, N \varepsilon)$. Then, if $\operatorname{dist}(b, M[1]) \leq \varepsilon$, not only do $M[1+N \varepsilon]$ and $K(B(N \varepsilon))$ intersect, but their intersection contains $M[1+N \varepsilon] \cap B(p,(N-1) \varepsilon)$ for some $p \in M[1]$ and $\|b-p\| \leq \varepsilon$. If in addition $\|b\| \leq 1, B(p,(N-1) \varepsilon) \subseteq B(1+N \varepsilon)$ and so by Lemma 7.2

$$
\operatorname{vol}_{2 d}(M[1+N \varepsilon] \cap K(B(N \varepsilon))) \geq \theta_{2 d} \cdot((N-1) \varepsilon)^{2 d},
$$

and so

$$
\begin{aligned}
& \int \operatorname{vol}_{2 d}(M[1+N \varepsilon] \cap K(B(N \varepsilon))) d K \\
& \geq \int_{\| \operatorname{dist}(b, M[1]) \leq \varepsilon} \theta_{2 d}((N-1) \varepsilon)^{2 d} d K \\
& =\theta_{2 d}((N-1) \varepsilon)^{2 d} \int \operatorname{dist} \underset{\| b, M[\leq 1}{(b, 1] \leq \varepsilon)} d K \\
& =O_{2 N-1} \cdots O_{1} \cdot \theta_{2 d}((N-1) \varepsilon)^{2 d} \cdot \operatorname{vol}_{2 N}(T(M, \varepsilon)),
\end{aligned}
$$

where we have used [26, Eq. 12.11].

Combining (7.13) and (7.14) yields

$$
\begin{aligned}
\operatorname{vol}_{2 N}(T(M, \varepsilon)) \leq \frac{N^{2 N}}{(N-1)^{2 d}} \cdot s \cdot \operatorname{deg}(M) \cdot \theta_{2 N} \cdot(1+N \varepsilon)^{2 d} \cdot \varepsilon^{2(N-d)} \\
=\left(\frac{N}{N-1}\right)^{2 N-2} \cdot N^{2} \cdot(N-1)^{2(N-d-1)} \cdot s \cdot \operatorname{deg}(M) \\
\quad \cdot \theta_{2 N} \cdot(1+N \varepsilon)^{2 d} \varepsilon^{2(N-d)} \\
\leq e^{2} \cdot N^{2} \cdot(N-1)^{2(N-d-1)} \cdot s \cdot \operatorname{deg}(M) \cdot \theta_{2 N} \cdot(1+N \varepsilon)^{2 d} \varepsilon^{2(N-d)}
\end{aligned}
$$

as desired.

Dividing by $\theta_{2 N}$ immediately yields

THEOREM 7.11. Let $M$ be a purely $2 d$-dimensional complex variety in $\mathbf{C}^{N}$. Let $s$ be defined as in Lemma 7.10. Then

$$
f(M, \varepsilon) \leq e^{2} N^{2}(N-1)^{2(N-d-1)} \cdot s \cdot \operatorname{deg}(M) \cdot(1+N \varepsilon) \cdot \varepsilon^{2(N-d)} .
$$

The upper bounds in Theorem 4.1 follow immediately from Theorem 7.11. This completes our proofs of upper bounds.

Note that for complex homogeneous varieties the upper bound in Theorem 7.11 is proportional to $\operatorname{deg}(M)$ whereas the lower bound in Theorem 7.7 is independent of $\operatorname{deg}(M)$. This gap is unavoidable as the following example shows. Suppose $M$ consists of $\operatorname{deg}(M)$ hyperplanes $H_{i}$ through the origin and tilted just slightly away from one another. For any $\varepsilon_{0}>0$ the hyperplanes may be chosen so close together that the set of points within $\varepsilon_{0}$ of any one overlaps almost completely with the set of points within $\varepsilon_{0}$ of any other. Therefore, for all $\varepsilon \geq \varepsilon_{0}$ the volume of $T(M, \varepsilon)$ will be insignificantly larger than the volume of $T\left(H_{i}, \varepsilon\right)$. Thus, no lower bound can be proportional to $\operatorname{deg}(M)$. In this example, $M$ is reducible; since irreducible homogeneous polynomials in at least three variables are dense in the set of all homogeneous polynomials in at least three variables and of the same degree, we see that restricting to irreducible varieties would not improve the lower bound. For 
nonhomogeneous complex varieties the lower bound in Theorem 7.7 is proportional to $1 / \operatorname{deg}(M)$; we conjecture that this factor may be removed.

We now turn to an asymptotic expression for $\operatorname{vol}_{2 N}(T(M, \varepsilon))$ when $M$ is homogeneous. The expression, not surprisingly, will simply be $\operatorname{vol}_{2 d}(M[1]) \cdot \theta_{2 N-2 d} \varepsilon^{2(N-d)}$, just as if $M[1]$ were a "rectangle" and $T(M, \varepsilon)$ a "rectangular parallelepiped" of radius $\varepsilon$.

To prove this, we will need some notation. If $M$ is a smooth $d$-manifold in $\mathbf{R}^{N}$, let

$$
T^{\prime}(M, \varepsilon)=\bigcup_{p \in M} N(p, \varepsilon)
$$

where $N(p, \varepsilon)$ is a closed $(N-d)$-ball of radius $\varepsilon$ centered at $p \in M$ and orthogonal to $M$. We will call $T^{\prime}(M, \varepsilon)$ a tubular neighborhood if each $v \in T^{\prime}(M, \varepsilon)$ lies in exactly one $N(p, \varepsilon)$. If, for example, $M$ is a hypersurface, $T^{\prime}(M, \varepsilon)$ will be a tubular neighborhood if $\varepsilon$ is smaller than the radius of curvature at all points of $M$.

Our main tool is due to Weyl [33]:

THEOREM 7.12. If $M$ is a closed d-dimensional manifold in $\mathbf{R}^{N}$ and $T^{\prime}(M, \varepsilon)$ is a tubular neighborhood of $M$, then

$$
\operatorname{vol}_{N}\left(T^{\prime}(M, \varepsilon)\right)=\sum_{k=N-d}^{N} c_{k, N}(M) \varepsilon^{k}
$$

where

$$
c_{N-d, N}(M)=\operatorname{vol}_{d}(M) \cdot \theta_{N-d} .
$$

The reason we cannot apply Weyl's theorem directly to varieties (which would have saved a great deal of effort!) is that varieties may have singularities, so that $T^{\prime}(M, \varepsilon)$ may never be a tubular neighborhood for any $\varepsilon$. If we eliminate a small neighborhood of the singular part of a variety, we may apply Weyl's theorem to the remainder. If we can show that the contribution to $\operatorname{vol}_{N}(T(M, \varepsilon))$ of this neighborhood of the singular part goes to 0 as the radius of the neighborhood shrinks to 0 , then the first term of Weyl's theorem will provide the correct asymptotic behavior of $\operatorname{vol}_{N}(T(M, \varepsilon))$ for small $\varepsilon$.

The proof goes as follows. Let $M$ be a homogeneous purely $2 d$-dimensional complex variety in $\mathbf{C}^{N}$. Let $S(M, r)=\{x \in M: \operatorname{dist}(x, S(M)) \leq r\}$ be an $r$ neighborhood in $M$ of the singular part of $M$. As usual, $S(M, r)[s]$ will denote $S(M, r) \cap \bar{B}(s)$. Note that $\lim _{r \rightarrow 0} \operatorname{vol}_{2 d}(S(M, r)[s])=0$. Let $M_{\text {in }}(r)=S(M, r)[1]$ and $M_{\text {out }}(r)=\left\{x \in M[1]: x \notin M_{\text {in }}(r)\right\}$. Note that $\lim _{r \rightarrow 0} \operatorname{vol}_{2 d}\left(M_{\text {out }}(r)\right)=$ $\operatorname{vol}_{2 d}(M[1])$.

Now for all $r$ we may apply Weyl's theorem to $M_{\text {out }}(r)$ : There is some $\varepsilon(r)>0$ such that when $\varepsilon<\varepsilon(r), T^{\prime}\left(M_{\text {out }}(r), \varepsilon\right)$ is a tubular neighborhood. We estimate $\operatorname{vol}_{2 N}(T(M, \varepsilon))$ as follows:

$$
\begin{aligned}
& \operatorname{vol}_{2 N}\left(T^{\prime}\left(M_{\text {out }}(r)[1-\varepsilon], \varepsilon\right)\right) \leq \operatorname{vol}_{2 N}(T(M, \varepsilon)) \\
& \quad \leq \operatorname{vol}_{2 N}\left(T^{\prime}\left(M_{\text {out }}(r), \varepsilon\right)\right)+\operatorname{vol}_{2 N}\left(T\left(M_{\text {in }}(r), \varepsilon\right)\right) .
\end{aligned}
$$

The bounds on $\operatorname{vol}_{2 N}(T(M, \varepsilon))$ hold because $T^{\prime}\left(M_{\text {out }}[1-\varepsilon], \varepsilon\right) \subseteq T(M, \varepsilon)$ and $T(M, \varepsilon) \subseteq T^{\prime}\left(M_{\text {out }}(r), \varepsilon\right) \cup T\left(M_{\text {in }}(r), \varepsilon\right)$. For fixed $r$ we have by Weyl's theorem

$$
\lim _{\varepsilon \rightarrow 0} \frac{\operatorname{vol}_{2 N}\left(T^{\prime}\left(M_{\text {out }}(r), \varepsilon\right)\right)}{\operatorname{vol}_{2 N}\left(T^{\prime}\left(M_{\text {out }}(r)[1-\varepsilon], \varepsilon\right)\right)}=\lim _{\varepsilon \rightarrow 0} \frac{\operatorname{vol}_{2 d}\left(M_{\text {out }}(r)\right)+O(\varepsilon)}{\operatorname{vol}_{2 d}\left(M_{\text {out }}(r)\right)+O(\varepsilon)}=1 \text {. }
$$


It remains to prove that for small enough $r, \operatorname{vol}_{2 N}\left(T\left(M_{\text {in }}(r), \varepsilon\right)\right)$ is negligible compared to $\operatorname{vol}_{2 N}\left(T^{\prime}\left(M_{\text {out }}(r), \varepsilon\right)\right)$. More precisely, we need to show that for all $\eta>0$ there is an $\bar{r}>0$ such that for all $r<\bar{r}$ and $\varepsilon<\varepsilon(r)$

$$
\frac{\operatorname{vol}_{2 N}\left(T\left(M_{\text {in }}(r), \varepsilon\right)\right)}{\operatorname{vol}_{2 N}\left(T^{\prime}\left(M_{\text {out }}(r), \varepsilon\right)\right)}<\eta \text {. }
$$

We will prove (7.17) by showing that for $N \varepsilon<r<1$,

$$
\begin{aligned}
& \operatorname{vol}_{2 N}\left(T\left(M_{\mathrm{in}}(r), \varepsilon\right)\right) \\
& \quad \leq e^{2} N^{2}(N-1)^{2(N-d-1)} \cdot \frac{\theta_{2 N}}{\theta_{2 d}} \cdot \operatorname{vol}_{2 d}(S(M, 2 r)[2]) \cdot \varepsilon^{2(N-d)} .
\end{aligned}
$$

Since for small $r$ and $\varepsilon<\varepsilon(r), \operatorname{vol}_{2 N}\left(T^{\prime}\left(M_{\text {out }}(r), \varepsilon\right)\right)$ behaves like $c \cdot \varepsilon^{2(N-d)}$, where $c$ is a constant near $\theta_{2(N-d)} \cdot \operatorname{vol}_{2 N}(M[1])$, the expression in (7.17) is bounded above by a constant times $\operatorname{vol}_{2 d}(S(M, 2 r)[2])$, which goes to 0 as $r$ goes to 0 .

We will prove (7.18) using the same technique as in Lemma 7.10. Let $d K=$ $d A \wedge d b$ be the kinematic density for the group of Euclidean motions. Then [26, Eq. 15.20] implies

$$
\begin{aligned}
& \int \operatorname{vol}_{2 d}(S(M, 2 r)[1+N \varepsilon] \cap K(B(N \varepsilon))) d K \\
& \quad=O_{2 N-1} \cdots O_{1} \cdot \operatorname{vol}_{2 N}(B(N \varepsilon)) \cdot \operatorname{vol}_{2 d}(S(M, 2 r)[1+N \varepsilon]) .
\end{aligned}
$$

Note that $\operatorname{vol}_{2 N}(B(N \varepsilon))=\theta_{2 N}(N \varepsilon)^{2 N}$ and, since $N \varepsilon<1$,

$$
\operatorname{vol}_{2 d}(S(M, 2 r)[1+N \varepsilon]) \leq \operatorname{vol}_{2 d}(S(M, 2 r)[2]) .
$$

Now if $\operatorname{dist}\left(b, M_{\text {in }}(r)\right) \leq \varepsilon$ and $\|b\| \leq 1$, then $\exists p \in M_{\text {in }}(r) \subseteq S(M, 2 r)[1+N \varepsilon]$ such that $\|b-p\| \leq \varepsilon$. Furthermore, since $K(B(N \varepsilon))=B(b, N \varepsilon)$,

$$
S(M, 2 r)[1+N \varepsilon] \cap B(p,(N-1) \varepsilon) \subseteq S(M, 2 r)[1+N \varepsilon] \cap B(b, N \varepsilon)
$$

and so by Lemma 7.2 and the fact that $N \varepsilon<r$,

$$
\operatorname{vol}_{2 d}(S(M, 2 r)[1+N \varepsilon] \cap K(B(N \varepsilon))) \geq \theta_{2 d} \cdot((N-1) \varepsilon)^{2 d} .
$$

From (7.19) we have therefore that

$$
\begin{aligned}
& \theta_{2 d} \cdot((N-1) \varepsilon)^{2 d} \int \operatorname{dist}\left(b, M_{\text {in }}(r)\right) \leq \varepsilon d A \wedge d b \\
& \leq O_{2 N-1} \cdots O_{1} \cdot \theta_{2 N}(N \varepsilon)^{2 N} \cdot \operatorname{vol}_{2 d}(S(M, 2 r)[2])
\end{aligned}
$$

or

$$
\theta_{2 d} \cdot((N-1) \varepsilon)^{2 d} \operatorname{vol}_{2 N}\left(T\left(M_{\mathrm{in}}(r), \varepsilon\right)\right) \leq \theta_{2 N}(N \varepsilon)^{2 N} \cdot \operatorname{vol}_{2 d}(S(M, 2 r)[2]),
$$

yielding (7.18) as desired.

In summary, we have

THEOREM 7.13. If $M$ is a purely $2 d$-dimensional complex variety in $\mathbf{C}^{N}$, then for asymptotically small $\varepsilon$ Weyl's theorem correctly estimates $\operatorname{vol}_{2 N}(T(M, \varepsilon))$. In particular, when $M$ is homogeneous,

$$
\operatorname{vol}_{2 N}(T(M, \varepsilon))=\operatorname{deg}(M) \cdot \theta_{2 d} \theta_{2(N-d)} \cdot \varepsilon^{2(N-d)}+o\left(\varepsilon^{2(N-d)}\right) .
$$

Proof. From Lemma 7.1 we have $\operatorname{vol}_{2 d}(M[1])=\operatorname{deg}(M) \cdot \theta_{2 d}$. 
COROLLARY 7.14. If $M$ is a homogeneous, purely $2 d$-dimensional complex variety in $\mathbf{C}^{N}$, then asymptotically

$$
f(M, \varepsilon)=\left(\begin{array}{l}
N \\
d
\end{array}\right) \cdot \operatorname{deg}(M) \cdot \varepsilon^{2(N-d)}+o\left(\varepsilon^{2(N-d)}\right) .
$$

This completes the proof of Theorem 4.1.

It appears that Ocneanu's proof of Theorem 4.3 may be modified so as to prove Conjecture 4.15 in an analogous way as Theorem 7.13: The contribution to the volume of $T(M, \varepsilon)$ from points near the singular part of $M$ is asymptotically negligible compared to the points away from the singular part, to which we can apply Weyl's theorem.

Now we turn to using these bounds to estimate the probability distribution $\operatorname{Prob}(\operatorname{dist}(p, M) \leq \varepsilon)$ when $M$ is homogeneous and $p$ is uniformly distributed on the unit sphere. We will use the following geometric interpretation of this probability: Let $T_{S}(M, \varepsilon)=\{p:\|p\|=1, \operatorname{dist}(p, M) \leq \varepsilon\}$; then, if the ambient space is $\mathbf{R}^{N}$, we have by definition

$$
\operatorname{Prob}(\operatorname{dist}(p, M) \leq \varepsilon)=\frac{\operatorname{vol}_{N-1}\left(T_{S}(M, \varepsilon)\right)}{O_{N-1}} .
$$

Now define $T_{C}(M, \varepsilon)=\{p: 0<\|p\| \leq 1, \operatorname{dist}(p /\|p\|, M) \leq \varepsilon \vee p=0\}$. Then $T_{C}(M, \varepsilon)$ is the intersection of a homogeneous set with $\bar{B}(1)$, and its intersection with the unit circle is $T_{S}(M, \varepsilon)$. Clearly,

$$
\begin{aligned}
\operatorname{vol}_{N}\left(T_{C}(M, \varepsilon)\right) & =\int_{0}^{1} \operatorname{vol}_{N-1}\left(T_{S}(M, \varepsilon)\right) r^{N-1} d r \\
& =\frac{\operatorname{vol}_{N-1}\left(T_{S}(M, \varepsilon)\right)}{N}
\end{aligned}
$$

so

$$
\operatorname{Prob}(\operatorname{dist}(p, M) \leq \varepsilon)=\frac{\operatorname{vol}_{N}\left(T_{C}(M, \varepsilon)\right)}{\theta_{N}},
$$

i.e., the fraction of the volume of the unit ball occupied by $T_{C}(M, \varepsilon)$. Since $T_{C}(M, \varepsilon) \subseteq T(M, \varepsilon)$,

$$
\operatorname{Prob}(\operatorname{dist}(p, M) \leq \varepsilon) \leq \frac{\operatorname{vol}_{N}(T(M, \varepsilon))}{\theta_{N}}=f(M, \varepsilon)
$$

and any upper bound for $f(M, \varepsilon)$ is an upper bound for $\operatorname{Prob}(\operatorname{dist}(p, M) \leq \varepsilon)$. This proves the upper bounds in Theorems 4.2 and 4.5.

Now we turn to asymptotic expressions. Suppose $M$ is homogeneous, $d$-dimensional and embedded in $\mathbf{R}^{N}$. From our previous discussion, it suffices to consider tubular neighborhoods and ignore singularities. In fact, we can do a purely local analysis by writing both $T(M, \varepsilon)$ and $T_{C}(M, \varepsilon)$ as the union of disjoint sections orthogonal to $M$, and comparing the volumes of the sections. In particular, for each $p \in M,\|p\|=1$, let $N(p, \varepsilon)$ be the section of $T(M, \varepsilon)$ whose "bases" are $(N-d)$-balls $\bar{B}( \pm p, \varepsilon)$ orthogonal to $M$ and which consists of all line segments connecting a point $p_{1} \in \bar{B}(p, \varepsilon)$ to a point $p_{2} \in \bar{B}(-p, \varepsilon)$. Let $N_{C}(M, \varepsilon)$ be the section of $T_{C}(M, \varepsilon)$ with the same bases and consisting of all line segments connecting $p_{1} \in \bar{B}(p, \varepsilon)$ and 
$-p_{1}$. Thus

$$
T(M, \varepsilon)=\bigcup_{\substack{p \in M \\\|p\|=1}} N(p, \varepsilon) \text { and } T_{C}(M, \varepsilon)=\bigcup_{\substack{p \in M \\\|p\|=1}} N_{C}(p, \varepsilon)
$$

Let $d p$ be a volume element of $M_{S}=\{p \in M,\|p\|=1\}$, and $N(d p, \varepsilon)$ and $N_{C}(d p, \varepsilon)$ be the union of the corresponding sections. Then, if $S(r)$ is the sphere of radius $r$,

$$
\begin{aligned}
\operatorname{vol}_{N}(N(d p, \varepsilon)) & =2 \int_{0}^{1} \operatorname{vol}_{N-1}(N(d p, \varepsilon) \cap S(r)) d r \\
& =2 \int_{0}^{1} \operatorname{vol}_{N-1}(N(d p, \varepsilon) \cap S(1)) r^{d-1} d r \\
& =2 \operatorname{vol}_{N-1}(N(d p, \varepsilon) \cap S(1)) / d
\end{aligned}
$$

and

$$
\begin{aligned}
\operatorname{vol}_{N}\left(N_{C}(d p, \varepsilon)\right) & =2 \int_{0}^{1} \operatorname{vol}_{N-1}\left(N_{C}(d p, \varepsilon) \cap S(r)\right) d r \\
& =2 \int_{0}^{1} \operatorname{vol}_{N-1}\left(N_{C}(d p, \varepsilon) \cap S(1)\right) r^{N-1} d r \\
& =2 \operatorname{vol}_{N-1}(N(d p, \varepsilon) \cap S(1)) / N
\end{aligned}
$$

i.e., the volume of the section of $T(M, \varepsilon)$ is $N / d$ times the volume of the section of $T_{C}(M, \varepsilon)$. Therefore,

$$
\lim _{\varepsilon \rightarrow 0} \frac{f(M, \varepsilon)}{\operatorname{Prob}(\operatorname{dist}(p, M) \leq \varepsilon)}=\lim _{\varepsilon \rightarrow 0} \frac{\operatorname{vol}_{N}(T(M, \varepsilon))}{\operatorname{vol}_{N}\left(T_{C}(M, \varepsilon)\right)}=\frac{N}{d},
$$

which proves the asymptotic results in Theorem 4.2 and (4.19) (the latter result depends on the truth of conjecture (4.15)).

Finally, we consider lower bounds on $\operatorname{Prob}(\operatorname{dist}(p, M) \leq \varepsilon)$. We will show

$$
\operatorname{vol}_{N}(T(M, \varepsilon)) \leq \operatorname{vol}_{N-1}\left(T_{S}(M, \varepsilon)\right)
$$

which will immediately imply

$$
\begin{aligned}
f(M, \varepsilon) & =\frac{\operatorname{vol}_{2 N}(T(M, \varepsilon))}{\theta_{N}} \leq \frac{\operatorname{vol}_{2 N-1}\left(T_{S}(M, \varepsilon)\right)}{\theta_{N}} \\
& =N \cdot \operatorname{Prob}(\operatorname{dist}(p, M) \leq \varepsilon) .
\end{aligned}
$$

To prove (7.20), consider the volume element $d x$ of $T_{S}(M, \varepsilon)$. Let $p \in M$ be a point closest to $x$, and construct the cylinder with base $d x$, axis parallel to the segment $\overline{0 p}$ and of length 1 . Since the length of the cylinder is 1 and its cross section is at most $d x$, its volume is at most $d x$. The union of all these cylinders fills up $T(M, \varepsilon)$ (perhaps multiply), so integrating their volumes over $T_{S}(M, \varepsilon)$ yields (7.20). (7.21) shows that $f(M, \varepsilon) / N$ is a lower bound on $\operatorname{Prob}(\operatorname{dist}(p, M) \leq \varepsilon)$ and completes the proofs of Theorems 4.2 and 4.5 . 
Computer Science Department

Courant Institute of Mathematical Sciences

New York University

251 Mercer Street

New York, New York 10012

1. V. I. ARNOL'D, "On matrices depending on parameters", Russian Math. Surveys, v. 26, 1971, pp. $29-43$.

2. E. H. BAREISS \& J. L. BARlow, Probabilistic Error Analysis of Floating Point and CRD Arithmetics, Dept. of Electrical Engineering and Computer Science, Northwestern University, Report 81-02-NAM-01, 1981.

3. J. W. Demmel, A Numerical Analyst's Jordan Canonical Form, Dissertation, Computer Science Division, University of California, Berkeley, 1983.

4. J. W. DEMMEL, "On condition numbers and the distance to the nearest ill-posed problem," Numer. Math., v. 51, 1987, pp. 251-289.

5. C. ECKART \& G. YOUNG, "The approximation of one matrix by another of lower rank", Psychometrika, v. 1, 1936, pp. 211-218.

6. H. FEDERER, Geometric Measure Theory, Springer-Verlag, Berlin and New York, 1969.

7. G. H. Golub \& C. F. VAN LoAn, Matrix Computations, Johns Hopkins Univ. Press, Baltimore, MD, 1983.

8. A. GRAY, "An estimate for the volume of a tube about a complex hypersurface," Tensor (N. S.), v. 39, 1982, pp. 303-305.

9. A. GRAY, "Comparison theorems for the volumes of tubes as generalizations of the Weyl tube formula," Topology, v. 21, 1982, pp. 201-228.

10. P. A. GRIFFITHS, "Complex differential and integral geometry and curvature integrals associated to singularities of complex analytic varieties," Duke Math. J., v. 45, 1978, pp. 427-512.

11. R. W. Hamming, "On the distribution of numbers," Bell System Tech. J., v. 49, 1970, pp. 1609-1625.

12. H. Hotelling, "Tubes and spheres in $n$-spaces, and a class of statistical problems," Amer. J. Math., v. 61, 1939, pp. 440-460.

13. D. Hough, Explaining and Ameliorating the Ill Condition of Zeros of Polynomials, Thesis, Mathematics Department, University of California, Berkeley, CA, 1977.

14. W. KAHAN, "Numerical linear algebra," Canad. Math. Bull., v. 9, 1966, pp. 757-801. (Gastinel's theorem appears here.)

15. W. KahaN, Conserving Confluence Curbs Ill-Condition, Technical Report 6, Computer Science Dept., University of California, Berkeley, August 4, 1972.

16. T. Kato, Perturbation Theory for Linear Operators, Springer-Verlag, Berlin and New York, 1966.

17. K. Kendig, Elementary Algebraic Geometry, Springer-Verlag, Berlin and New York, 1977.

18. D. E. KNUTH, The Art of Computer Programming, Vol. 2, Addison-Wesley, Reading, Mass., 1969.

19. E. Kostlan, Statistical Complexity of Numerical Linear Algebra, Dissertation, Math. Dept., University of California, Berkeley, 1985.

20. P. LELONG, Fonctions plurisousharmoniques et formes différentielles positives, Gordon and Breach, Paris, 1968.

21. A. OCNEANU, On the Volume of Tubes About a Real Variety, unpublished report, Mathematical Sciences Research Institute, Berkeley, 1985.

22. A. OCNeAnu, On the Loss of Precision in Solving Large Linear Systems, Technical Report, Mathematical Sciences Research Institute, Berkeley, 1985.

23. J. RENEGAR, "On the efficiency of Newton's method in approximating all zeros of systems of complex polynomials," Math. Oper. Res., v. 12, 1987, pp. 121-148.

24. J. R. RICE, "A theory of condition," SIAM J. Numer. Anal., v. 3, 1966, pp. 287-310.

25. A. RuhE, "Properties of a matrix with a very ill-conditioned eigenproblem," Numer. Math., v. 15,1970 , pp. 57-60.

26. L. A. SANTALó, Integral Geometry and Geometric Probability, Encyclopedia of Mathematics and Its Applications, Vol. 1, Addison-Wesley, Reading, Mass., 1976.

27. S. SMALE, "The fundamental theorem of algebra and complexity theory," Bull. Amer. Math. Soc. (N. S.), v. 4, 1981, pp. 1-35. 
28. S. SMALE, "Algorithms for solving equations," presented at the International Congress of Mathematicians, Berkeley, 1986.

29. G. W. STEWART, "Error and perturbation bounds for subspaces associated with certain eigenvalue problems," SIAM Rev., v. 15, 1973, p. 752.

30. G. Stolzenberg, Volumes, Limits, and Extensions of Analytic Varieties, Lecture Notes in Math., vol. 19, Springer-Verlag, Berlin and New York, 1966.

31. P. R. ThiE, "The Lelong number of a point of a complex analytic set," Math. Ann., v. 172, 1967, pp. 269-312.

32. B. L. VAN DER WAERden, Modern Algebra, Vol. 1, Ungar, New York, 1953.

33. H. WEYL, "On the volume of tubes," Amer. J. Math., v. 61, 1939, pp. 461-472.

34. J. H. Wilkinson, Rounding Errors in Algebraic Processes, Prentice-Hall, Englewood Cliffs, N.J., 1963.

35. J. H. Wilkinson, The Algebraic Eigenvalue Problem, Oxford University Press, Oxford, 1965. 36. J. H. WILKINSON, "Note on matrices with a very ill-conditioned eigenproblem," Numer. Math, v. 19, 1972, pp. 176-178.

37. J. H. WILKINSON, "On neighboring matrices with quadratic elementary divisors," Numer. Math., v. 44, 1984, pp. 1-21.

38. J. H. WILKINSON, "Sensitivity of eigenvalues," Utilitas Math., v. 25, 1984, pp. 5-76. 